\title{
Coding Encrypted Messages Into Music
}

\author{
by
}

Vinay Réginald Kumar, B.Math.

\author{
A thesis submitted to \\ the Faculty of Graduate Studies and Research \\ in partial fulfilment of the requirements for the degree of
}

\section{Masters of Science}

School of Mathematics and Statistics

Ottawa-Carleton Institute of Mathematics and Statistics

\author{
Carleton University \\ Ottawa, Ontario, Canada
}

August 2006

(C) Copyright 2006

Vinay Réginald Kumar 


$\begin{array}{ll}\begin{array}{l}\text { Library and } \\ \text { Archives Canada }\end{array} & \begin{array}{l}\text { Bibliothèque et } \\ \text { Archives Canada }\end{array} \\ \begin{array}{l}\text { Published Heritage } \\ \text { Branch }\end{array} & \begin{array}{l}\text { Direction du } \\ \text { Patrimoine de l'édition }\end{array} \\ \begin{array}{l}\text { 395 Wellington Street } \\ \text { Ottawa ON K1A 0N4 }\end{array} & \begin{array}{l}\text { 395, rue Wellington } \\ \text { Ottana ON K1A ON4 } \\ \text { Canada Oa }\end{array}\end{array}$

Your file Votre référence ISBN: 978-0-494-18366-3 Ourfile Notre référence ISBN: 978-0-494-18366-3

NOTICE:

The author has granted a nonexclusive license allowing Library and Archives Canada to reproduce, publish, archive, preserve, conserve, communicate to the public by telecommunication or on the Internet, loan, distribute and sell theses worldwide, for commercial or noncommercial purposes, in microform, paper, electronic and/or any other formats.

The author retains copyright ownership and moral rights in this thesis. Neither the thesis nor substantial extracts from it may be printed or otherwise reproduced without the author's permission.
AVIS:

L'auteur a accordé une licence non exclusive permettant à la Bibliothèque et Archives Canada de reproduire, publier, archiver, sauvegarder, conserver, transmettre au public par télécommunication ou par l'Internet, prêter, distribuer et vendre des thèses partout dans le monde, à des fins commerciales ou autres, sur support microforme, papier, électronique et/ou autres formats.

L'auteur conserve la propriété du droit d'auteur et des droits moraux qui protège cette thèse. $\mathrm{Ni}$ la thèse ni des extraits substantiels de celle-ci ne doivent être imprimés ou autrement reproduits sans son autorisation.
In compliance with the Canadian

Privacy Act some supporting forms may have been removed from this thesis.

While these forms may be included in the document page count, their removal does not represent any loss of content from the thesis.
Conformément à la loi canadienne sur la protection de la vie privée, quelques formulaires secondaires ont été enlevés de cette thèse.

Bien que ces formulaires aient inclus dans la pagination, il n'y aura aucun contenu manquant. 


\section{Acknowledgements}

There are many people that have helped me with my work. I thank my thesis supervisor, Dr. Daniel Panario for his patience and guidance. To Dr. Brett Stevens and Dr. François Théberge, I thank them for their constructive criticism as well as many helpful ideas. Many thanks to Dr. James Wright and Jack Coghill for the numerous hours explaining basic music concepts to me, but also for their advice and suggestions to steer me in the right direction. To Valerie Daley, I cannot say thank

you enough. Finally, I would like to thank my parents, Irène and Virender, for their support and being there for me.

Thank you. Merci. Dhanyavaad. 
To my grandmother, Sumitra Devi Gupta, for her inspiration. 


\section{Abstract}

This thesis develops a method that uses music to encode and decode any encrypted message. We begin with a brief survey of past research on this topic. Afterwards, we present our own initial attempts that have failed. The first attempt uses a twodigit representation and the second uses functions to convert messages into music. Improving on past research and overcoming our own flaws is the motivation for Chapter 4. It is there where we achieve our goal: provide an algorithm to encode and decode any encrypted message into music that 'makes sense', that is, music that is audibly pleasant. Using basic ideas from linear algebra, messages are converted into vectors, and we perform matrix-vector multiplication to encrypt. The resulting vector has its entries converted into music. To ensure that the generated music makes sense we use the pentatonic scale. A decryption process, also based in linear algebra, allows us to recover the original message. We observe that any available cryptosystem may be used instead of the one proposed here, since the music encoding remains the same. The purpose of including music is to convince 'enemies' that the intercepted message does not contain any hidden information. 


\section{Contents}

Acknowledgements i

Dedication ii

Abstract

1 Introduction 1

2 Related Issues $\quad 4$

2.1 A Relationship of Numbers and Music . . . . . . . . . . . 4

2.2 Various Musical Encoding Schemes . . . . . . . . . . . . . 5

3 Initial Attempts $\quad 11$

3.1 Two-Digit Representation Code . . . . . . . . . . . . . . . . 12

3.2 Encrypting Messages As Functions . . . . . . . . . . . . . . 24

4 A Simpler Approach $\quad 30$

iv 
4.1 Some Basic Music Theory . . . . . . . . . . . . . . . . 30

4.2 The Matrix Code . . . . . . . . . . . . . . . . . . . 35

4.3 Summary of Required Algorithms . . . . . . . . . . . . . . 56

4.4 Remarks and Examples . . . . . . . . . . . . . . 62

4.5 Coding Numbers Into Music . . . . . . . . . . . . . 79

5 Conclusions $\quad 88$

$\begin{array}{lr}\text { Bibliography } & 90\end{array}$ 


\section{Chapter 1}

\section{Introduction}

At one moment or another we have all heard about the similarities between mathematics and music. The Greeks discovered that certain ratios with respect to the length of two strings produced pleasing sounds. For example, if the ratio between the length of the two strings is $2: 3$, then we call this pitch perfect fifth. Should the ratio be 3:4, then we call this pitch perfect fourth. Further information may be found at [15], to see what many others explored based on what the Greeks started. Now, we see that certain relationships exist between mathematics and musical intervals, but can we do more?

We know that sending secret messages has often been a priority. Whether it was during the great wars of the last century or back to over two thousand years in the past, many procedures to send encrypted messages have been developed [11]. On 
one hand we have the desire to explore how mathematics and music are related while on the other hand we have this need to send secret messages. This leads us to ask: can we send secret messages with music? The Merriam-Webster Online Dictionary defines music as:

the science or art of ordering tones or sounds in succession, in combination, and in temporal relationships to produce a composition having unity and continuity [19].

In other words, the music should be reasonably pleasant to the ear.

In this thesis we attempt to provide methods to encode encrypted messages into music. In Chapter 2, we begin our discussion by briefly examining related ideas that have been thought of over the last few centuries. As we will see, there have been various attempts. In Chapter 3 , we present our ideas that failed. The codes could not guarantee that we would obtain music. Moreover, certain ambiguities in the decoding phase are present and need to be resolved. In the first code, we assign numerical values to all permissible symbols in a special way. We then translate these numbers into music. The second code also translates numbers into music. However, we propose using functions. In Chapter 4 we present a code that resolve some of the flaws from previous ideas and still generate music. In this main chapter, we use simple concepts from linear algebra to encrypt. First we convert a message into a large 
number. This number is then transformed into a vector. Second, we perform matrix multiplication to obtain a new vector, which is then coded into music. We make use of the pentatonic scale to guarantee that we obtain music. The decryption process is outlined and uses matrix operations from linear algebra. We do not prove that the proposed encryption process is secure. Our interests lie in the coding process. As such, any cryptosystem (RSA, AES and so on) may be used and do not influence the 'coding into music' part of the procedure. To the best of our knowledge, this method is an innovation. Finally, in Chapter 5 we review the main results and give further problems to research related to using music to encode messages.

We note that the intended receiver need not have to listen to the music. Essentially, we recognize that music can be sent through various media. For example, one can physically burn the music on a compact disc and hand it to the receiver or the music can be sent in an e-mail as an attachment. If 'Alice' sends a message to 'Bob', he knows that she will be sending some music in a 'special' format. Without the proper software to decode and decrypt, all that the enemy has is music, should the message be intercepted. Since the music makes sense one may simply discard it and not realize that there is some hidden information. The music is only there to convince others that they have not intercepted a secret message. 


\section{Chapter 2}

\section{Related Issues}

The exchange of secret information has a long history and it would be impossible to explore it fully. In this chapter we briefly discuss what has been done so far in the area of creating music via numbers and secret messages. As we see later on, although music is applied in the coding process, certain coding processes do not yield what one would necessarily call music. Our goal is to present the motivation for this thesis: to create a coding process that will encode encrypted messages into (pleasant) music.

\subsection{A Relationship of Numbers and Music}

Combining mathematics with music is not a new idea. A quick Internet search reveals that successful attempts have been made. One such example is found in [16]. 
At this site, various numbers, functions and even the Pascal Triangle have been used to create music. The idea is to uniquely assign a particular pitch, tone, etc. to a digit or group of digits. By no means is this a cryptosystem. However, one can be motivated to develop a code that takes encrypted messages and changes them into music. For our intentions, we cannot apply the same process, as it would become quite cumbersome and overly complicated. After all, we cannot plan for every possible combination of digits. We have that an individual creates a message and a string of digits representing the message is produced and subsequently encrypted. The last thing we want is to have a long series of 'if-then' statements. Nonetheless, the site offers some insight and inspiration to help develop the code.

\subsection{Various Musical Encoding Schemes}

It would seem that many ventured into the realm of amalgamating music and ciphers $[2,9,21,28]$. One of the earlier attempts involved the creation of a grid, where five out of seven notes can be one out of four letters and the remaining two notes can be one out of three letters. In this ' $7 \times 4$ ' system, one can 'compose' someone's name for example. With this particular coding scheme, the encoding process is quite simple. It is the decoding process which is quite problematic: we receive a note and depending which one it is, all we know is that it can be one out of three or one 
out of four letters. In other words, it is similar to that of a telephone keypad: the number ' 2 ' for example can be used to represent the letters $a, b$ and $c$.

Wilkins [21] and Terzi [28] proposed a different method to use music. Each letter was assigned a particular note in a particular octave. Thus an 'A' in one octave would represent the letter ' $k$ ' for example, while an ' $A$ ' in a higher octave would represent the letter ' $t$ '. This coding process addresses the problem of the previous coding scheme. However, it has two design flaws: the music that the code creates would not necessarily be pleasant and even if the key could be kept secret from potential enemies, one could still use certain standard attacks upon intercepting the message, such as performing a 'frequency analysis'. That is to say, one could count how many times each note is being used and compare the count with a table ordering letters from the most commonly used to the least commonly used. For example, in the English language the letter $e$ is the most commonly used. Thus, one would suspect that the most commonly used note is representing the letter $e$. Eventually, one should be able to determine which note represents which letter.

In Kircher's Musurgia (Rome 1650), the proposed coding scheme is based on using various orchestral instruments. The idea does have the same flaw as Wilkins' and Terzi's scheme. It is nonetheless a different approach. In Kircher's 'coding' process, we would have the following table: 
Table 2.2.1

\begin{tabular}{|c|c|c|c|c|}
\hline Number of notes played in succession & 1 & 2 & 3 & 4 \\
\hline First instrument & a & b & c & d \\
\hline Second instrument & e & f & g & h \\
\hline Third instrument & i & k & l & m \\
\hline Fourth instrument & n & o & p & q \\
\hline Fifth instrument & r & s & t & v \\
\hline Sixth instrument & w & x & y & z \\
\hline
\end{tabular}

P. G. Schott and H. a Sunde had proposed systems similar to Kircher's in their respective works Schola steganographia (Nuremberg 1665) and Steganologia (Nuremberg - no date). In 1596, Porta had also devised a similar system by way of ringing various bells. Should a city be under siege, one could send a message by ringing 'Bell 1' once for the letter 'A', twice for ' $\mathrm{B}$ ' and thrice for ' $\mathrm{C}$ '. Then, one would ring 'Bell 2' once for ' $\mathrm{D}$ ' and so on. Perhaps the most elaborate coding scheme resembling these is due to Michael Haydn. In his Biographische Skizze von Michael Haydn (Salzburg 1808), Haydn developed a complex system that even included the use of rests (or pauses) to denote various kinds of punctuation [9].

In the fifteenth century the work Tractatus varii medicinales used a system of five different pitches. Each pitch varied in five different ways, thus giving twenty-five 
symbols. The pitches could be varied either by note values or stem-directions. Philip II of Spain had employed a similar system, circa 1560. Devised in 1596, the papal cryptographic service was a more complicated system; using nine different pitches, each variable in eight ways. This allowed for seventy-two symbols $[2,9]$.

There is a major setback to these coding schemes: for $21^{\text {st }}$ century cryptographic utility a code should be able to create any message. This means that not only uppercase and lowercase letters, but all ninety-five different symbols must be at our disposal. (The number ninety-five comes from the fact that there are ninety-five printable ASCII characters [31].)

Facing this problem, it would seem that the best way to involve music in codes is to simply apply steganography. This practice is defined below.

Definition 2.2.1 Steganography is the art and science of writing hidden messages in such a way that no one apart from the intended recipient knows of the existence of the message; this is in contrast to cryptography, where the existence of the message itself is not disguised, but the content is obscured [35].

Steganography is a clever approach that has existed for a long time and is still being used today. Now that one can easily send 'mp3' and 'wav' files through e-mails or by various other means, steganography has applications beyond art or messages 
written with lemon juice ${ }^{1}$. The following websites $[23,24,25,30]$ explain how one would go about applying music to steganography. A particular website [18] has links to download software that can perform steganography. Hence, we may still use this procedure to hide information.

Figure 2.1:
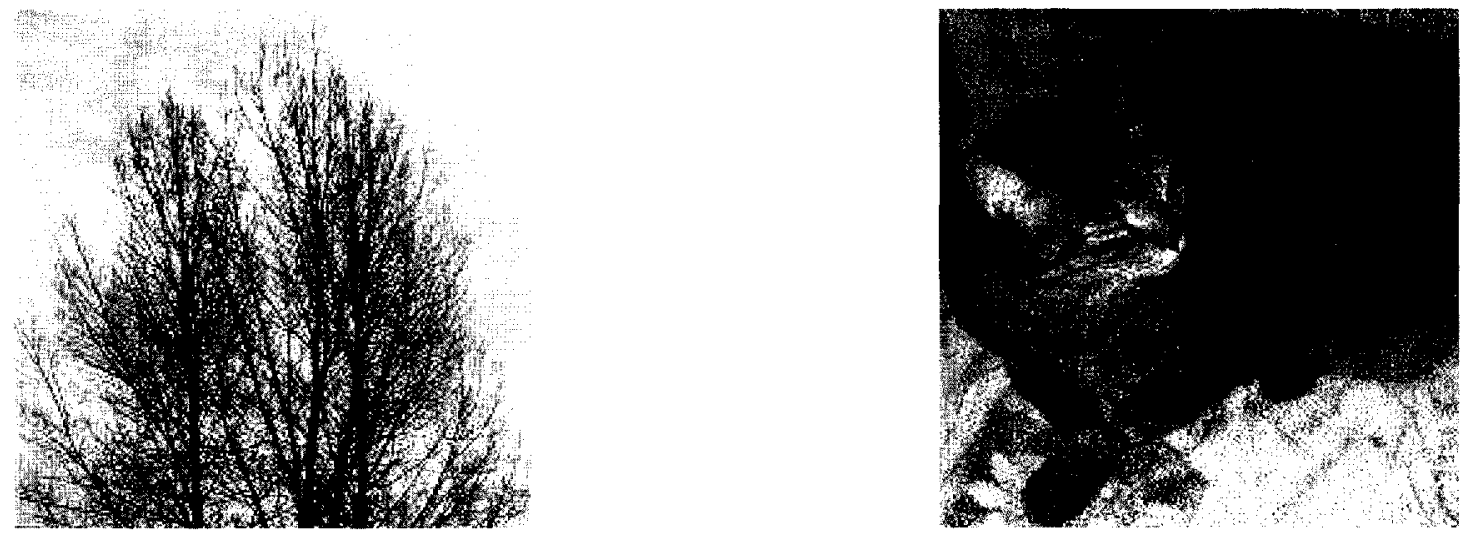

Example 2.2.1 Figure 2.1 above is an example of steganography, where the image that is sent is a tree, but the hidden image is that of a cat. What has been done is quite simple. The last two bits of each colour component have been removed, thus making the image a little brighter [35]. For a musical example, we found one on the Internet, where [25] is a part of a song containing a hidden message, while [24] is

\footnotetext{
${ }^{1}$ The process is shown at [22]. Essentially one writes a message on a piece of paper, where the 'ink' is the lemon juice. Once dried, the paper will appear to be blank. However, when the paper is heated the message will reappear.
} 
the same part of the song unaltered.

The goal of this thesis is not to use steganography. What we want is to determine if one can actually send music as the message and not use music as the carrier. Unlike the ideas of some of the composers we have discussed above, our goal is not limited to just coding messages into random pitch or chord sequences. We want this music to have musical sense, that is to say, the music should have proper musical structure. 


\section{Chapter 3}

\section{Initial Attempts}

In this chapter we show various attempts to code numbers into music. We say attempts, because these codes do have certain flaws either mathematically or musically. By mathematical flaw we mean to say that the code could easily be broken. However, because of these flaws we are able to create a final and hopefully sound mathematical code, which also makes musical sense and is not cacophonic. For our final system, see Chapter 4 . Before we begin our discussion, we state the following definitions.

Definition 3.0.1 A sharp, denoted by $\sharp$, raises the natural note while a flat, denoted by $b$ lowers the natural note.

Definition 3.0.2 A triad is a chord consisting of three notes which can be arranged 
to form two superimposed thirds. Using the notation $\mathrm{X}-\mathrm{Y}-\mathrm{Z}, \mathrm{X}$ is the root note, $\mathrm{Y}$ is a major or minor third above $\mathrm{X}$, and $\mathrm{Z}$ is a perfect 5 th above $\mathrm{X}$ (i.e., a major or minor third above $\mathrm{Y}$ ). So the C-major triad is C-E-G [10].

\subsection{Two-Digit Representation Code}

The first step to create any kind of code is to establish which symbols are permissible. As stated previously, we would like to allow all ninety-five printable ASCII characters in any message. Our assumption here is that anyone could use the code and so messages of every type should be permissible. We further note that linguistic messages are written in English.

Secondly, after one has created a message, how will it be encoded? In this coding method we assign a two-digit number to each symbol. Here by two-digit we also include the numbers 0 through 9 where these numbers have a leading 0 , so that $01=$ 1 and so forth. Of course, we have one hundred such numbers and only ninety-five are required. However, we do not discard the remaining five numbers. Characters such as spaces, commas, periods and the letter $e$ are potentially the symbols that will be used most frequently in the messages and so it makes sense for such symbols to have more than one representation. Consider the following table representing each symbol uniquely by a two-digit number: 
Table 3.1.1

\begin{tabular}{|c|c||c|c||c|c|}
\hline symbol & integer & symbol & integer & symbol & integer \\
\hline $\mathrm{A}$ & 03 & $\mathrm{q}$ & 38 & $\sharp$ & 59 \\
\hline $\mathrm{B}$ & 04 & space & 47 & $@$ & 98 \\
\hline
\end{tabular}

We note that Table 3.1 .1 is a small portion of possible pairings between symbols and numbers.

Thirdly, we now need a way to convert the string of numbers into music. We consider a table that helps us link these numbers musically. In [7] one finds the following table:

\section{Table 3.1.2}

\begin{tabular}{|c|c|c|c|c|c|c|c|}
\hline & $\mathrm{I}$ & ii & iii & IV & $\mathrm{V}$ & vi & vii $^{\circ}$ \\
\hline $\mathrm{I}$ & $\mathrm{X}$ & $\mathrm{X}$ & $\mathrm{X}$ & $\mathrm{X}$ & $\mathrm{X}$ & $\mathrm{X}$ & $\mathrm{X}$ \\
\hline ii & & $\mathrm{X}$ & & $\mathrm{X}$ & $\mathrm{X}$ & & $\mathrm{X}$ \\
\hline iii & & $\mathrm{X}$ & $\mathrm{X}$ & & & $\mathrm{X}$ & \\
\hline $\mathrm{IV}$ & $\mathrm{X}$ & & & $\mathrm{X}$ & $\mathrm{X}$ & & $\mathrm{X}$ \\
\hline $\mathrm{V}$ & $\mathrm{X}$ & & & & $\mathrm{X}$ & & \\
\hline vi & & $\mathrm{X}$ & & $\mathrm{X}$ & & $\mathrm{X}$ & \\
\hline vii & $\mathrm{X}$ & & $\mathrm{X}$ & & & & $\mathrm{X}$ \\
\hline
\end{tabular}


We make some notes about the table and its notation, i.e., the significance of the roman numerals used in Table 3.1.2.

1. The roman numerals indicate the scale-degree upon which the triad is built in some scale. For example, in the scale of $\mathrm{C}, \mathrm{C}$ is represented by I. However, in the scale of $\mathrm{G}, \mathrm{C}$ is represented by IV. We use these numerals to avoid using the phrase: 'in the scale of.'

2. The 'uppercase numerals' indicate major triads. These are abbreviated by ' $M$ '. We may often omit using ' $M$ ' for simplicity whenever the context is clear. The 'lowercase numerals' indicate minor triads. These are abbreviated by ' $\mathrm{m}$ '. Lastly, the triad with a superscript ' 0 ' indicates that this triad is diminished ${ }^{1}$. Thus, 'DM' is the D major triad and therefore, writing D is equivalent to DM; 'Em' is the $\mathrm{E}$ minor triad; $\mathrm{F} \sharp^{\circ}$ is the $\mathrm{F} \sharp$ diminished triad.

3. In Table 3.1.2 an $\mathrm{X}$ means that in harmonic triad progressions a 'row triad $i$ ' leads to a 'column triad $j$ ', which we will abbreviate by: $i \mapsto j$.

4. The 'I' triad is known as the tonic triad. In other words, if we are in the key of ' $\mathrm{G}$ ', $\mathrm{I}=\mathrm{GM}$ and in the key of ' $\mathrm{C}$ ', $\mathrm{I}=\mathrm{CM}$.

5. The table is an abstraction. Thus, depending on the key we are in (or what the tonic triad is), a particular triad has a different roman numeral value. For

\footnotetext{
${ }^{1} \mathrm{~A}$ diminished triad has a minor third and diminished fifth.
} 
example, in the key of $\mathrm{G}, \mathrm{IV}=\mathrm{CM}$, but in the key of $\mathrm{F}, \mathrm{V}=\mathrm{CM}$.

Of course, Table 3.1.2 gives us a basic framework, allowing us to make good triad progressions. This table, however, is a simplistic view and so further researching various triad progressions would be required to determine its accuracy. For example, Table 3.1.2 claims that we cannot play D-Em, a common progression. Nonetheless, assuming that we work only with it, we then create another table. The table that follows assigns digits to the triads:

\section{Table 3.1.3}

\begin{tabular}{|c|c|c|c|c|c|c|c|}
\hline Roman numeral notation & I & ii & iii & IV & V & vi & vii $^{\circ}$ \\
\hline Key of G & G & Am & Bm & C & D & Em & F $^{\circ}$ \\
\hline Play once & 4 & 1 & 3 & 0 & 7 & 2 & 6 \\
\hline Play twice & 2 & 8 & 9 & 1 & 0 & 5 & 3 \\
\hline
\end{tabular}

Table 3.1 .3 is merely an illustration and the values can be altered to whichever one desires. We now make a few remarks about the Table 3.1.3.

1. The first row gives us a general form. In other words, regardless of the key the digits correspond to a given major or minor triad. The second row gives us the actual triads in the arbitrarily chosen key of G major. This is only there to help visualize, we are by no means restricted to the key of $\mathrm{G}$. 
2. If we wish to access the digit 2 , we apparently have two triads at our disposal: $\mathrm{G}$ and Em (or in general notation: I and vi) - which is true. However, accessing it from the Em triad requires us to play the triad once as it is in the first of the last two rows, while accessing it from the $G$ triad requires us to play it twice, because it is in the last row. This can be modified in the sense that instead of actually playing the triads, we would have to hold the G triad twice as long as the Em.

3. Keeping note 2 in mind, suppose we have the following string of triads: G-GG. This always means the number 24 and never 42 , or 444 . In other words, it is always assumed that if a triad is repeated a number of times consecutively, we will always be in the second row of that triad, i.e., G-G-G-G-G $=224$.

So how do we combine all this? If one quickly looks at Table 3.1.3, we realize that upon 'composing' a message, we may have to play two (or more) triads that do not, theoretically sound well together. To solve this problem we create 'transition triads'.

Definition 3.1.1 By transition triads, we mean that between two triads $X$ and $Y$, we insert a transition triad $T$ such that the triad progressions $X, T$ and $T, Y$ are audibly pleasing.

How many such triads do we need? 
Lemma 3.1.1 In the graph that follows, from any triad at most three intermediate triads are required to reach the ' $T$ ' triad.

Figure 3.1:

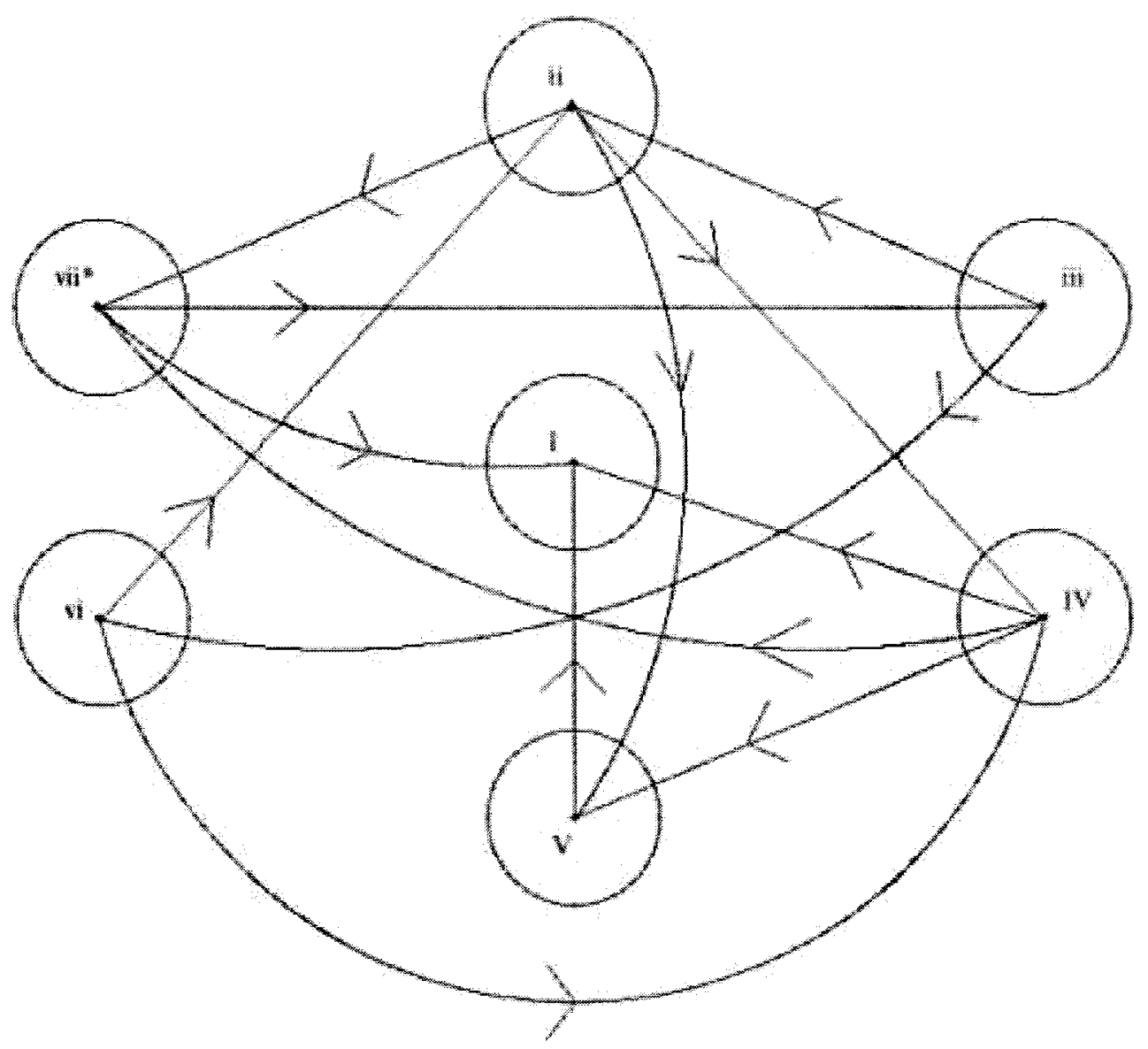

Proof. The graph is an alternate way to look at Table 3.1 .2 , omitting self-loops and the paths of the form ' $\mathrm{I} \mapsto j$ for all triads $j$. We simply proceed by enumerating all possible paths that lead to the I tonic triad, such that the lengths are less than or equal to three, summarized below: 


$$
\begin{aligned}
& \triangleright \text { ii } \mapsto\{I V \text { or } V\} \mapsto I \Rightarrow \text { length is } 2 . \\
& \triangleright \text { iii } \mapsto \text { ii } \mapsto\{I V \text { or } V\} \mapsto I \Rightarrow \text { length is } 3 \text { (alternatively, iii } \mapsto \text { vi } \mapsto I V \mapsto I \Rightarrow \\
& \text { length is } 3 \text { ). } \\
& \triangleright \mathrm{IV} \mapsto \mathrm{I} \Rightarrow \text { length }=1 \text {. } \\
& \triangleright \mathrm{V} \mapsto \mathrm{I} \Rightarrow \text { length }=1 \text {. } \\
& \triangleright \text { vi } \mapsto \mathrm{IV} \mapsto \mathrm{I} \Rightarrow \text { length }=2 . \\
& \triangleright \text { vii } \mapsto \mathrm{I} \Rightarrow \text { length }=1 .
\end{aligned}
$$

We note that there are other possible paths, however, the point was only to show that starting from any triad, we can reach the tonic triad I in less than or equal to three triads. The fact that other paths exists will be applied upon the creation of the transition triads, in the development of the code.

A necessary step in the encryption process is to determine what are the encrypted values of the transition triads. Of course, one naturally asks: why are we concerned about the encrypted values of the transition triads? When we are creating the message, we do not care if it makes musical sense prior to encryption, that is, only the coded message should make musical sense. Thus, the transition triads only have importance after the message has been coded. To determine what the values of transitions triads are we use the following procedure. 
1. We have three unknown numbers, say $r, s$ and $t$. The three numbers are the images of some other two-digit numbers. Moreover, the two digits that compose them are such that some (or all) of the triads that represent the first digit can lead into some (or all) of the triads that represent the second digit.

2. Once $r, s$ and $t$ have been determined, we decrypt them into say $R, S$ and $T$ respectively. These latter three numbers are now 'reserved' and so the remaining numbers are available for the ASCII characters.

To help illustrate this process, we provide an example and again we refer the reader to Tables 3.1.2 and 3.1.3. We also observe that by Lemma 3.1.1, we have that we may need up to three transition triads. This means that we now require a total of 98 triads for the 95 ASCII characters and the three transition triads.

\section{Example 3.1.1}

- Let $i$ and $j$ be triads such that $i \mapsto j$. Let $i$ represent digit $d_{1}$ and $j$ represent digit $d_{0}$. Then, the number $n=d_{1} d_{0}$ is the image of a transition triad. To illustrate, let us compute the transition triads $r, s$ and $t$.

1. Let $r=81$. Using Table 3.1.3, 8 can be obtained by playing Am-Am and 1 is obtained by playing either Am or C-C, that is, when the key is not specified, 8 is obtained via ii-ii and 1 is obtained via ii or IV-IV. Since ii $\mapsto$ IV, we have satisfied the requirement above and so $r=81$. 
This means that, based on Table 3.1.2, any triad that naturally leads to Am can use this set of triads as a transition triad. Since Am is ii, we look for entries of column ii. This is the sequence of triads, which end with either G, Am, Bm or Em. Moreover, this transition triad also has the power of leading to the sequence of triads beginning with $G, C, D, F \sharp^{\circ}$. We would then have to decrypt $r$ to $R$ to 'reserve' the number $R$ as a transition triad when construction a table like Table 3.1.1.

2. Next, we find a number for $s$. We need to make sure that $s$ leads to at least one triad different from those of $r$. Furthermore, we need a different set of triads that lead to $s$. Consider the number 74 . We obtain 74 by D-G and by applying Tables 3.1.2 and 3.1.3 we observe that $7 \mapsto 4$. Thus, letting $r=74$, we notice that any sequence of triads ending with G, Am, C and D lead to D. Furthermore, 74 leads to every other triad, as 4 is represented by $G$, where $G$ is $\mathrm{I}$ in the key of G. Thus, $s=74$.

We would then have to decrypt $s$ to $S$ to 'reserve' the number $S$ as a transition triad.

3. Thus far, the only sequence of triads, which we are missing are those that end on $\mathrm{F}^{\circ}$. This means that for $t$ we need to consider those triads that naturally lead out of $F \sharp^{\circ}$. Those triads are $\mathrm{G}, \mathrm{Bm}$, and of course $\mathrm{F} \sharp^{\circ}$. We have the 
added constraint that when looking at each digit, we require that the digits can lead to one another, otherwise we would need a transition triad within the transition triad. What is a good candidate for $t$ then? We select 33, which can be obtained by $F \sharp^{\circ}-F \sharp^{\circ}-F \sharp^{\circ}-F \sharp^{\circ}$ and $F \sharp^{\circ}-F \sharp^{\circ}-B m$. It would seem that we could obtain 33 from Bm-Bm. However, we remind the reader that by convention, playing $\mathrm{Bm}-\mathrm{Bm}$ represents the digit nine. We would then have to decrypt $s$ to $S$ to 'reserve' the number $S$ as a transition triad, where $s=33$. We summarize all this information below.

$\triangleright$ The transition triads are: 33 (obtained by: $F \sharp^{\circ}-F \sharp^{\circ}-F \sharp^{\circ}-F \sharp^{\circ}$ or $F \sharp^{\circ}-F \sharp^{\circ}-B m$ ), 74 (obtained by: D-G) and 81 (obtained by: Am-Am-Am or Am-Am-C-C).

$\triangleright$ If the letter ends on $G(4,2)$, no transition required.

$\triangleright$ If the letter ends on $A m(1,8)$, use 33,74 or 81 .

$\triangleright$ If the letter ends on $\mathrm{Bm}(3,9)$, use 81 .

$\triangleright$ If the letter ends on $\mathrm{C}(0,1)$, use 33 or 74 .

$\triangleright$ If the letter ends on $\mathrm{D}(7,0)$, use 74 .

$\triangleright$ If the letter ends on $\operatorname{Em}(2,5)$, use 74 or 81 .

$\triangleright$ If the letter ends on $F \sharp^{\circ}(6,3)$, use 33 . 
$\triangleright$ If the letter begins on $\mathrm{G}(4,2)$, use $33\left(\mathrm{~F} \sharp^{\circ}-\mathrm{F} \sharp^{\circ}-\mathrm{F} \sharp^{\circ}-\mathrm{F} \sharp^{\circ}\right.$ only), 74 or 81 (AmAm-C-C only).

$\triangleright$ If the letter begins on $\mathrm{Am}(1,8)$, use 33 (only $\mathrm{F} \sharp^{\circ}-\mathrm{F} \sharp^{\circ}-\mathrm{Bm}$ ) or 74 .

$\triangleright$ If the letter begins on $\operatorname{Bm}(3,9)$, use 33 ( $\mathrm{F} \sharp^{\circ}-\mathrm{F} \sharp^{\circ}-\mathrm{Bm}$ only), 74 .

$\triangleright$ If the letter begins on $\mathrm{C}(0,1)$, use 74 or 81 .

$\triangleright$ If the letter begins on $\mathrm{D}(7,0)$, use 74 or 81 .

$\triangleright$ If the letter begins on $\operatorname{Em}(2,5)$, use 33 (only $F \sharp^{\circ}-F \sharp^{\circ}-B m$ ), 74 .

$\triangleright$ If the letter begins on $F \sharp^{\circ}(6,3)$, use 33 ( $F \sharp^{\circ}-F \sharp^{\circ}-F \sharp^{\circ}-F \sharp^{\circ}$ only), 74 or 81 .

* For simplicity we did not use roman numerals to help the reader visualize a little more clearly as to what was going on. In this example, we were working in the key of $\mathrm{G}$, i.e., $\mathrm{I}=\mathrm{G}$. This means that other keys yield alternate tables, similar to Table 3.1.3. Finally, we note that using Tables 3.1 .2 and 3.1 .3 we can check that as $F \sharp^{\circ}$ does not lead into either the Am or C triads, then 61 cannot be used as a transition triad.

Now, there are a few problems. Perhaps the most obvious one is the fact that if one were to send a message containing thirty characters (a relatively short message), then we would require sixty triads. A possible solution is to create some sort of shift$k e y$, whereby common words or parts of words such as 'the' or 'ght' could have their 
own two-digit representation, thus reducing the number of triads used. We would have to create a second table, similar to Table 3.1.1 and to access it we would have a number in Table 3.1.1, say 47, that would have to be 'typed'. However any long message could not be sent by this coding process, since to decrease the number of triads we need to increase the number of tables, and thus increase the amount of information to keep in memory.

A second problem is that we have seven triads and ten digits. This means that only three digits are going to be repeated, which is quite limiting. If we look at Table 3.1 .3 again, the repeated digits are 0,1 and 3 . What if the encrypted message uses few of these digits? Then, we are not using the 'power of repetition'. A way around this is to not look at digits over base 10 , but perhaps base 2 . We would then have a table that would be like the following.

Table 3.1.4

\begin{tabular}{|c|c|c|c|c|c|c|c|}
\hline Roman numeral notation & I & ii & iii & IV & V & vi & vii $^{\circ}$ \\
\hline Key of G & G & Am & Bm & C & D & Em & F $\sharp^{\circ}$ \\
\hline Play once & 0 & 1 & 0 & 1 & 0 & 1 & 0 \\
\hline Play twice & 1 & 0 & 1 & 0 & 1 & 0 & 1 \\
\hline
\end{tabular}

The advantages of using base 2 representation is that we eliminate the need of transition triads and we can actually use numbers up to 127 to represent various 
characters and symbols. However, to represent a number, we now need at least eight triads. To illustrate, let us say that we want to code the number 74 , represented as 01001010. Then, one could play for example G,C,D,G,C,D,C,D to represent 74 .

In spite of these problems, there is potentially some merit to this coding scheme. If one has a short message to send, then this code could be useful. For example, to send a particular string of integers of length say 40 , then this code could be employed. This idea is one that we may come in handy later on.

\subsection{Encrypting Messages As Functions}

The next code is inspired from a software application called 'Pure Data' [27], or PD for short. Exploring PD one finds that we can use functions, specifically polynomials, to generate music or at the very least a series of tones. The idea then came to devise a way to create a polynomial or a few polynomials that would contain the message. Essentially, the zeroes of the polynomial would contain the encrypted message. Before stating the coding procedure, we first need a definition that provided some added inspiration, which is due to Gödel ${ }^{2}$.

Definition 3.2.1 A Gödel number $g$ is created as follows.

1. First we establish what are all the permissible symbols. In our case, this

\footnotetext{
${ }^{2}$ Gödel used this special form of numbering to prove his famous Incompleteness Theorem [8].
} 
includes common phrases, words and parts of words. Call this set $\mathbb{S}$.

2. Second we assign a unique number $e$ to each $s \in \mathbb{S}$. Call this set $\mathbb{E}$.

3. Third we create a set of primes, denoted by $\mathbb{P}$.

4. A Gödel number is the number $g=p_{1}^{e_{1}} \times \ldots \times p_{t}^{e_{t}}$, where $e_{i} \in \mathbb{E}$ and $p_{j} \in \mathbb{P}$.

Since our messages resemble a Gödel number, we must encrypt each symbol through some process. One possible method is to use RSA encryption, which we outline and illustrate below.

Definition 3.2.2 The following procedure is known as the RSA Cryptosystem.

1. Pick two primes $p, q$ such that $n=p q$. Set $s=(p-1)(q-1)$. The message $m$ (converted into some positive integer) is divided into blocks bounded by some number $n$. The block sizes are equal to $2^{k}$, where $2^{k}<n \leq 2^{k+1}$. We have base 2 if we are working over $\mathbb{F}_{2}$.

2. Choose a number $e$, such that $3 \leq e \leq s$ and $e$ is relatively prime to $s$.

3. Compute $m^{e} \bmod n=c$, where $m$ is the plaintext and $c$ is the ciphertext.

4. To decode, find $d$ such that $e d \equiv 1 \bmod s$ and compute $c^{d} \bmod n$ to recover the plaintext $m$. 
Example 3.2.1 Suppose we choose the prime $p=101$, so that $s=101-1=100$. Let $e$ represent the encrypting exponent and say it is equal to 13 . We compute $13^{-1}$ modulo 100 and observing that $13 \times 77 \equiv 1 \bmod 100$ and so $d=77$ is the decrypting exponent. If we wish to send the message 'HELLO', we translate it into its numerical representation. For the purpose of this example, suppose that H,E,L and $\mathrm{O}$ are respectively represented as $08,05,12$ and 15 . Then encrypting is done as follows: $8^{13} \equiv 18 \bmod 101,5^{13} \equiv 56 \bmod 101,12^{13} \equiv 53 \bmod 101$ and $15^{13} \equiv 7$ mod 101. The encrypted message would then be: 18, 56, 53, 53 and 07 for $\mathrm{H}, \mathrm{E}$, $\mathrm{L}, \mathrm{L}$ and $\mathrm{O}$ respectively ${ }^{3}$. Upon receiving the message, one would only need to compute $18^{77}, 56^{77}, 53^{77}$ and $7^{77}$ all modulo 101 to recover the original message. This example is a simpler version of RSA encryption presented in Definition 3.2.2, since we only use one prime instead of two.

Our encoding and encryption procedures are summarized below.

1. Let $\mathbb{P}_{k}$ be the set of primes up to and including some prime number $k$. The primes are used to indicate the position of each character in the message. To illustrate, let $p_{1}, p_{2}, \ldots, p_{t} \in \mathbb{P}_{k}$, be the primes used in a message of length t. Let $e_{1}, e_{2}, \ldots, e_{t} \in \mathbb{E}$ be positive integer exponents. These exponents represent a character that can be used in the message, where the exponents are the numerical values to permissible symbols. Then, the message can then

\footnotetext{
${ }^{3}$ We would actually send the string 1856535307 .
} 
be read 'numerically' as:

$$
p_{1}^{e_{1}}, p_{2}^{e_{2}}, \ldots, p_{t}^{e_{t}}
$$

The $p_{i}^{e_{i}}$ are first mapped to some other integers and then become the roots of the polynomial(s) that we create in the fifth step.

2. Of the finite set $\mathbb{P}_{k}$, there is a maximal element $p_{\max }$. Then, there exists a prime larger than $p_{\max }$, which we denote as $p^{*}$. The prime $p^{*}$ is our encrypting prime. We first compute $s=p^{*}-1$. Then, we find integers $e$ and $d$, such that $e d \equiv 1 \bmod s$. The integer $e$ will be the encrypting exponent, while $d$ will serve to decrypt, as illustrated in Example 3.2.1. We further note that we can see how large $p^{*}$ must be. Since we must apply exponents $e_{i}$ to each $p_{i}$, we require that $p^{*}$ must be greater than $p_{\max }^{\max \left\{e_{1}, e_{2}, \ldots, e_{t}\right\}}$. This 'restriction' will ensure that if we need to encrypt the letter ' $A$ ', and it so happens that the required exponent is $\max \left\{e_{1}, e_{2}, \ldots, e_{t}\right\}$ AND the prime used happens

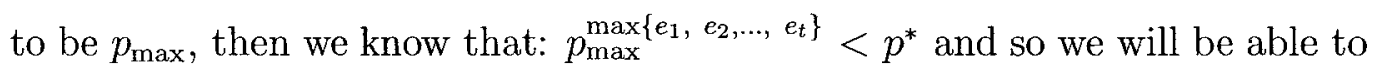
recover the letter ' $\mathrm{A}$ '.

3. We note that $\left|\mathbb{P}_{k}\right|<\infty$. We therefore require that this set be 'large enough'. In other words, if the longest message that we can write out is of length $l$, then $\left|\mathbb{P}_{k}\right|$ should be much larger than $l$. This is because we do not want to use all of our 'primes' in the message. For one message we may want to use only a 
particular subset of $\mathbb{P}_{k}$ and for another message we use a different subset.

4. The encryption is done at this step.

i. First, for every prime $p_{i}$ and its exponent $e_{i}$ we compute $\left(p_{i}^{e_{i}}\right)^{e} \bmod p^{*}$, for $1 \leq \mathrm{i} \leq l$.

ii. Next, we denote the encrypted $\left(p_{i}^{e_{i}}\right)^{e}$ as $\overline{\left(p_{i}^{e_{i}}\right)}$. Next, we apply a permutation $\Pi$ to each $\overline{\left(p_{i}^{e_{i}}\right)}$. In other words $\Pi\left(\overline{p_{i}^{e_{i}}}\right)=a_{i}$, for some integer $a_{i}$.

iii. To decrypt, we need only to first apply $\Pi^{-1}$ to each $a_{i}$ and then compute ${\overline{\left(p_{i}^{e_{i}}\right)}}^{d}\left(\bmod p^{*}\right)$ to obtain the original $p_{i}^{e_{i}}$.

5. To create our polynomials, we simply take each $a_{i}$ and create linear factors.

Therefore, our encrypted message will look like:

$$
f(x)=\prod_{j=1}^{k}\left(x-a_{i}\right)
$$

6. We then input $f(x)$ into PD to generate a single tone.

There are certain problems with this code.

1. The first problem stems from the fact that unlike Tchebychev polynomials that actually do generate pleasant tones ${ }^{4}$, our polynomials are for all intents

\footnotetext{
${ }^{4} \mathrm{PD}$ includes several audio examples and by testing these various programs, the assertion is readily verified.
} 
and purposes, random. Thus, we cannot guarantee that the tones we generate are pleasing to the ear. In some 'test cases', we created static noise. One could propose a way to 'smoothen' the sounds, however this means instructing a computer to distinguish between 'good' and 'bad' sounds, a concept that is far too complex for us to even consider.

2. Secondly, we observe that we may be generating an unpleasant sequence of tones or static instead of creating music. The goal of this thesis is to generate music. Unfortunately, as illustrated in the PD software, a sequence of 'pleasing tones' may be generated, however collectively, the 'music' it generates is not necessarily pleasing to the ear.

3. Lastly, the message would have to be somewhat short. This is seen simply by considering the size of $p^{*}$. The larger the size of $\mathbb{P}_{k}$, the faster $p^{*}$ grows. Moreover, it is quite time consuming searching for such large primes.

We have now seen two ways to code messages musically. While some of their flaws could possibly be resolved, there is one inescapable flaw: only very short messages could ever be sent using these methods. Despite the flaws we described, certain procedures within these coding processes were employed that do have some merit. It is these flaws and procedures that help develop the code we present in the next chapter. 


\section{Chapter 4}

\section{A Simpler Approach}

When tackling a problem, more often than not we tend to look for complicated solutions instead of going back to the basics. After exploring various ideas and concepts that had appeared to put us 'on the right track', we instead found ourselves with a new problem to solve, thus complicating an already complicated process. In this chapter, we simply ask ourselves: what is it that we want? Answering this tells us exactly how to solve the goal of this thesis.

\subsection{Some Basic Music Theory}

In Section 3.1 we looked at the possibility of using various triads to represent digits. We were then faced with numerous problems, which included: 
- the length of the musical work;

- the pleasantness of the music;

- the notion of transition triads.

The last two problems go hand in hand, as the latter seems to resolve the former to help create some sort of musical flow. However, we cannot necessarily guarantee that the music as a whole will sound like an actual composition with some kind of melody. In the end, we would end up with a sequence of triads that make musical sense, but it would be lacking certain musical coherences and complexities. Moreover, we assume that the message would be intercepted and so this coding scheme would easily give away that it is hiding some information.

The first of the problems above is the one that truly causes us to make us reconsider each coding scheme as a whole. If each digit is to be represented by at least two triads, then a message containing only twenty-five symbols (letters, numbers, punctuation marks, etc.) would require us to use at least fifty triads. Thus, if we only want to send brief messages - and by brief we mean to say a message of length not exceeding ten symbols, then we are fine. Nonetheless, a typical message is much longer and as such we need to drastically alter the coding process.

The first step we take is to forget about triads and simply look at individual notes. Next we ask: how does one compose music and what is most essential to 
music? Melody and harmony immediately come to mind. That is, we have a series of notes being played and then there is a background to help with the flow of sounds. If we consider a guitar solo in a typical rock-and-roll song, the lead guitarist plays a series of notes while the bass and drum players maintain the beat to accompany the solo. Of course, we also need to make sure that the music is ear pleasing.

However, cacophony does have a desirable quality for our purposes: randomness. Once a message has been converted numerically, at first sight, we have a somewhat random string of integers. Now, if we visualize the twelve keys on a piano that form one octave, ten of these would be the required digits. We then 'play' our number, obtaining a random string of notes. Let us consider an example.

Example 4.1.1 Consider the C-scale on a piano, that is, going left to right beginning at $\mathrm{C}$ and ending at the next occurrence of this note. Figure 4.1 that follows may help the reader visualize. Assign 0 to $\mathrm{C}, 1$ to $\mathrm{C}^{1}, 2$ to $\mathrm{D}, \ldots, 9$ to $\mathrm{A}$. We note that in this case, $A \sharp$ and $B$ do not have a numerical value. Now, suppose that the message is converted to the number 1746 . Then, musically, we would play the notes $\mathrm{C} \sharp, \mathrm{G}, \mathrm{E}, \mathrm{F} \sharp$.

Of course, the messages we send are numbers that are much larger than 1746. In fact, the message we send may be represented by a number (or numbers) having length exceeding one hundred. In the preceding example, the four notes have a

\footnotetext{
${ }^{1} \mathrm{C} \sharp$ is the black key immediately to the right of $\mathrm{C}$. The same key is also $\mathrm{Db}$.
} 
Figure 4.1:

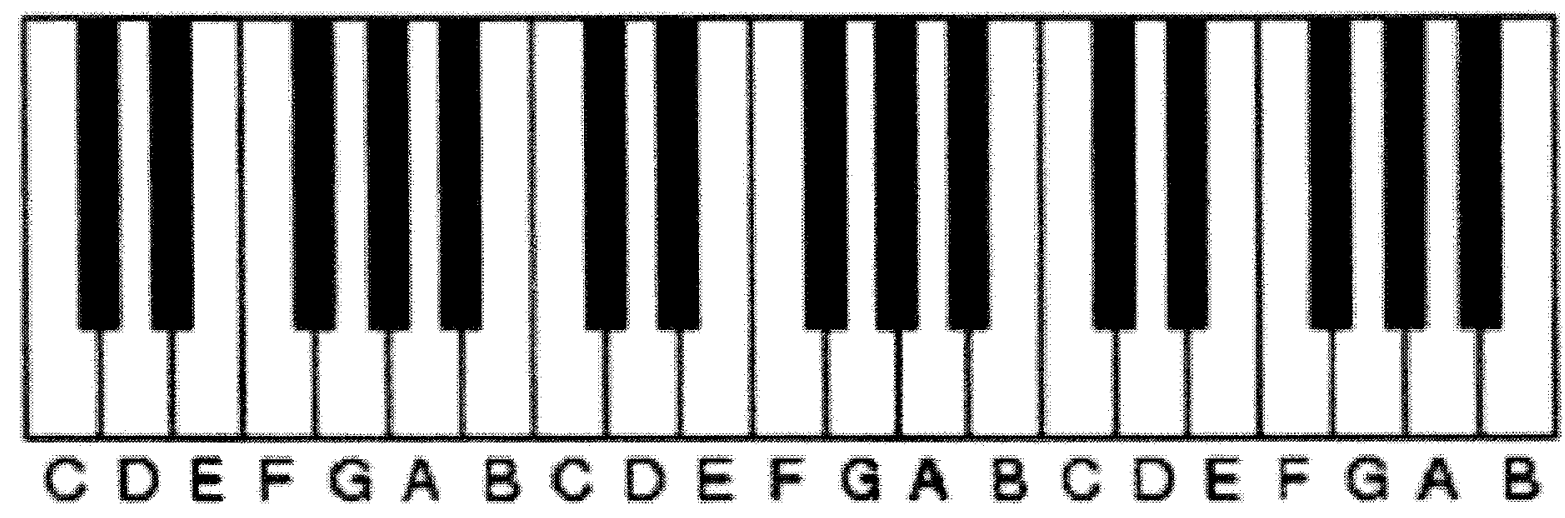

pleasing sound when played successively. In general however, this may not be the case if we were to have one hundred or more notes. The 'music' will sound like someone randomly playing notes without any regard to make the piece harmonious. So what do we do? Is there a scale that we can use that will allow us to not turn to transition triads or to 'smoothen' the music via some other means? In other words: is there a scale that has the quality of random being melodious? The answer is yes.

We look at a scale that is commonly used in music: the pentatonic scale. This scale is all around us, from guitar solos by many rock-and-roll bands to baby toys. In fact, it is used in many cultures [32]. Perhaps the main reason why it is so commonly used is the fact that this scale has one major quality: we can play any note in this scale in any order we choose and it sounds pleasant to the ear. 
This property is most beneficial to us since it eliminates our need of transition triads. However, why can we play any note in any order? What makes this scale so special? Simply put, the pentatonic scale does not contain any harsh sounds. The pentatonic scale ${ }^{2}$ lacks dissonances since it has no semitones or tritones. Moreover, the pentatonic scale is rich in consonances since it contains three major thirds, one minor third and four perfect fourths. These are defined below.

Definition 4.1.1 By seconds, thirds, fourths and so on, we mean the interval between two notes. For example, if we begin on $\mathrm{C}$, then $\mathrm{D}$ is the next note and so the interval between them is called a second. The interval between $\mathrm{C}$ and $\mathrm{E}$ is a third and so forth. The natural state of these intervals (in a major scale) is called a major interval. If we flatten one of the second, third, sixth or seventh notes, then we create a minor interval. Some intervals cannot be flattened and are so called perfect. These intervals are the fourths and fifths [7]. Finally, a semitone is a half step. That is, if we pick any key on a piano, the next key immediate to the left or right is a half step down or up respectively. Finally, a tritone is six semitones.

The pentatonic scale also gives us a bonus. Since we are using base 10 representation when writing numbers, we use two octaves of the pentatonic scale. Consider the following definitions and example to help illustrate.

\footnotetext{
${ }^{2}$ In Forte's set notation, this is the vector 5-35, summarized in [5] and [12].
} 
Definition 4.1.2 The pentatonic scale is composed of five notes of a given major scale. These notes are the first, second, third, fifth and sixth of the scale.

Definition 4.1.3 The circle of fifths is a progression where each chord (or note) is a fifth above the previous chord (or note) [7] or is a geometrical space that depicts relationships among the twelve tones of an octave [34].

Example 4.1.2 Using the circle of fifths, if we start on $\mathrm{C}$, then the pentatonic scale would be the following pitches: C, G, D, A, and E. Another way to view the pentatonic scale is the following: in the key of $\mathrm{G}$-flat $(\mathrm{Gb})$, the pentatonic scale is composed of all of the black keys on the piano.

\subsection{The Matrix Code}

In this section, we discuss how we can create an efficient code that involves music. The key to this code is Linear Algebra and the inspiration comes from the RSA encryption. The power of RSA is that there is no known method to factorize a number in a reasonable amount of time. What we want is some kind of property found in linear algebra, which is similar to this.

It is a known fact that the product of two matrices is not unique. As an example, consider: 
Example 4.2.1

$$
\left(\begin{array}{cc}
1 & 0 \\
0 & -1
\end{array}\right)\left(\begin{array}{cc}
2 & -3 \\
3 & -5
\end{array}\right)=\left(\begin{array}{rr}
2 & -3 \\
-3 & 5
\end{array}\right)=\left(\begin{array}{cc}
-1 & 0 \\
0 & -1
\end{array}\right)\left(\begin{array}{rr}
-2 & 3 \\
3 & -5
\end{array}\right) .
$$

We use this fact to help develop the code. Why? Should someone intercept our key matrix, only by guessing would the message matrix be retrieved by the enemy. At this point we have a message matrix $M$ and a encrypting matrix $C$ such that the product $C M=R$ is sent, where $R$ is the received matrix. To create the entries of $M$, we remind the reader of Gödel numbering since is used in our encryption process. However, this type of representation is not required to code the message, since more secure methods do exist. We only use it to present an alternate approach in encryption.

1. Establish what are all the permissible symbols, including common phrases, words and parts of words. Call this set $\mathbb{S}$.

2. Assign a unique number $e$ to each $s \in S$. Call this set $\mathbb{E}$.

3. Create a set $\mathbb{P}$ of primes, larger than maximal length of presumable message.

4. The message $m$ we create is of the form $m=p_{1}^{e_{1}} \ldots p_{t}^{e_{t}}$, where $e_{i} \in \mathbb{E}$ and $p_{j} \in \mathbb{P}$. 
* We note that in our encryption process if $i$ is less than $j$, this does not imply that $p_{i}$ is less than $p_{j}$.

Our message has now been converted into a large number. We first discuss how the matrix $M$ is obtained and later we explain how we create $C$. At this point, if we omit having to merge music into the code, the code simply reduces to the following.

1. Let $l=n^{2}$, where $C$ and $M$ are $n \times n$ matrices.

2. Let $m$ denote the message number as defined above. Thus, the (large) integer $m$ is of the form

$$
m=\sum_{k=0}^{l} m_{k} 10^{k} .
$$

We note that $m_{l}$ may be 0 , so that really $m$ is some integer of 'degree' $d \leq l$.

3. The $(i, j)^{\text {th }}$ entry of $M$, which we may simply denote as $m_{i j}$ or $\left[m_{i j}\right]$, is the coefficient $m_{k}$, such that

$$
k=n(i-1)+j-1
$$

This is obtained by noting that we have $i-1$ rows of length $n$ and there are $j-1$ columns. To arrive at this relation we are simply taking the number $m$ and breaking it into pieces of length $n$, noting that if $d<l$, then $m_{d+1}=\ldots=$ $m_{l}=0$. Next, the first piece of length $n$ is placed in the first row, placing the digits left to right. Thus, the $(1,1)$-entry is $m_{0}$, the $(1,2)$-entry is $m_{1}$ and so 
on. We continue until all $l$ digits have been placed. This verifies the relation above. Of course, one could interchange $i$ and $j$ so that we place the digits column-wise rather than row-wise. Hence, one is not limited to the relation above.

4. Next, we do the matrix multiplication $C M=R$, where in principle $C$ is any invertible matrix ${ }^{3}$ and send the matrix $R$. If $R$ is intercepted, it seems difficult to determine $M$ without knowing $C$ since matrix factorization is not unique. Essentially, for every $i$ and $j$, one would have to simultaneously solve equations of the form:

$$
\sum_{k=1}^{n} c_{i k} m_{k j}=r_{i j},
$$

where the only information that the attacker has is the $r_{i j}$ and may possibly know the structure of $C$ (this will be discussed later). Since matrix multiplication is not unique, the attacker may have several candidates for $C$ and $M$. Thus, if one does not possess a table similar to Table 3.1.1, then we cannot verify which pair of matrices $C$ and $M$ are being used.

In the process just illustrated, there are several issues. The first is that of 'rounding-errors'. There exists methods to compute inverses of matrices. However, depending on the size of the entries, computing an inverse may force one to approximate, something that we do not want. Consider the following example.

\footnotetext{
${ }^{3}$ We can easily guarantee that $C$ is invertible simply by how it is constructed.
} 


\section{Example 4.2.2}

$$
A \times A^{-1}=\left(\begin{array}{cc}
10^{100} & 0 \\
0 & 1
\end{array}\right)\left(\begin{array}{cc}
10^{-100} & 0 \\
0 & 1
\end{array}\right)=\left(\begin{array}{cc}
1 & 0 \\
0 & 1
\end{array}\right) .
$$

Depending on the program, the $(1,1)$-entry of $A^{-1}$ could be approximated to 0 . In this case, we would have the following product.

$$
A \times A^{\prime-1}=\left(\begin{array}{cc}
10^{100} & 0 \\
0 & 1
\end{array}\right)\left(\begin{array}{ll}
0 & 0 \\
0 & 1
\end{array}\right)=\left(\begin{array}{ll}
0 & 0 \\
0 & 1
\end{array}\right) \neq\left(\begin{array}{ll}
1 & 0 \\
0 & 1
\end{array}\right) .
$$

Thus, we would not obtain the inverse of $A$.

By the preceding example, we need some efficient way of obtaining $M$ and simply put, computing $C^{-1}$ is not feasible, especially in view of the shear dimension of the matrices, where, in our examples, our matrices will be $32 \times 32$. Another issue deals with how $M$ is created. By going 'digit by digit' to create $M$, it could be possible to devise certain attacks to obtain $M$ as its coefficients are elements of $\mathbb{Z}_{10}$. Lastly, how do we send $R$ ? Do we actually send $n^{2}$ integers? If we use $n=30$ say, this means that we are required to send 900 integers. Sending 900 integers can take too much time and perhaps take up too much memory.

Since we want to integrate music in the coding process, we need to reduce the number of integers. Otherwise, a message such as 'Meet me in the Herzberg Building by the pendulum at $3 h 14 \mathrm{~m} 15 \mathrm{~s}$ am' could potentially be a half-hour musical composition. Thus, if intercepted, one could easily guess that the music is a hidden message. 
So to reduce the number of integers we propose to decrease the dimension of $M$. In fact, the message will now be in vector form, $\vec{m}$, instead of matrix form ${ }^{4}$.

Now that we have covered this, let us now return to our encrypting process. Our encrypting matrix $C$ is of course, pardon the pun, the key. We need to make sure that we have a process that will make it easy for us to recover the message yet difficult if not impossible for the enemy. Moreover, we need to ensure that $C$ is invertible. Since the entries of $C$ are in $\mathbb{Z}$, one could appeal to the following result.

Lemma 4.2.1 If a matrix $A$ with integer entries has determinant \pm 1 , then its inverse also has integer entries.

Proof. Let us denote the adjoint matrix of $A$ as $\operatorname{adj}(A)$ and the determinant of $A$ as $\operatorname{det}(A)$. Then,

$$
A^{-1}=\frac{\operatorname{adj}(A)}{\operatorname{det}(A)}
$$

Since the entries of $A$ are integers, then so are the entries of $\operatorname{adj}(A)$. Moreover, as $\operatorname{det}(A)= \pm 1$, we immediately see that the entries of $A^{-1}$ are also integers.

This result would be useful if we have to compute $C^{-1}$. As $C$ has its entries over $\mathbb{Z}$, we need only to make sure that $\operatorname{det}(C)= \pm 1$. Hence, if upon computing the inverse decimals are returned, we then know that a rounding-error has occurred.

\footnotetext{
${ }^{4}$ Equation (4.2) can thus be simplified.
} 
However, the fact that $\operatorname{det}(C)= \pm 1$ is quite limiting and this piece of information could be quite useful when attacking the encrypted message. Thus, we only require that $C \in \mathrm{GL}(\mathbb{Z}, n)$.

Definition 4.2.1 $\mathrm{GL}(\mathbb{Z}, n)$ is the general linear group of $n \times n$ matrices over the integers, that is, $n \times n$ non-singular matrices over $\mathbb{Z}$.

As stated above, we do not want to compute the inverse of a matrix to decrypt a message. Instead, the shear size of $C$ (since $n$ will be of the order of at least thirty) tells us to consider using its $L U$ factorization. However, to make it a little more complicated, we also require the use of a permutation matrix $P$. We define these now.

Definition 4.2.2 Any matrix $A$ can be factorized as the product of two matrices $L$ and $U$, where the matrix $L$ is a lower triangular matrix and the matrix $U$ is an upper triangular matrix.

The product $A=L U$, however, is not unique.

Definition 4.2.3 A permutation matrix $P$ is a binary matrix that has exactly one entry 1 and 0 elsewhere.

Such a matrix has the following two key properties.

1. The inverse of a permutation matrix is equal to its transpose, i.e., $P^{T}=P^{-1}$. 
2. A permutation matrix has the property of permuting rows or columns. To obtain negative entries we need only to scale certain rows by -1 .

So, if we let $A=L U$, then $C=P A$. Since $P$ is on the left, then it permutes the rows of $A$. For us to decrypt a message $\vec{m}$, we follow the procedure below upon receiving the vector $\vec{r}$.

1. $C \vec{m}=P A \vec{m}=P L U \vec{m}=\vec{r}$.

2. $L U \vec{m}=P^{T} \vec{r}$.

3. Solve for $y$, where $L y=P^{T} \vec{r}$.

4. Now that we know $\vec{y}$, then we can easily solve for $\vec{m}$, where $U \vec{m}=y$.

We note that the entries of $\vec{m}$ need not be positive. If we denote the entries of $\vec{m}$ as $m_{i}$, then picking a sufficiently large positive number $s$ (where $s$ is a number that will be larger than any value that an entry of $\vec{m}$ can take in absolute value), then we calculate the Jacobi symbol of $m_{i}$ with respect to $s$, so that $m_{i} \mapsto\left(\frac{m_{i}}{s}\right) m_{i}$. It seems plausible that using the Jacobi symbol will not guarantee an 'even' distribution of negative and positive signs. We may expect that a random generator will give us more or less the same number of negative and positive signs. Thus, although using the Jacobi symbol is more expensive, it seems to give a less predictable distribution of plus and minus signs. 
Definition 4.2.4 If a number $m$ is a square modulo $s$, then it is a quadratic residue, otherwise it is a quadratic non - residue [4].

Definition 4.2.5 Let $p$ be an odd prime and $m$ be any number not divisible by $p$. Then, the Legendre symbol $\left(\frac{m}{p}\right)$ is defined by

$$
\left(\frac{m}{p}\right)=\left\{\begin{array}{c}
1 \text { if } m \text { is a quadratic residue } \bmod p \\
-1 \text { if } m \text { is a quadratic non }- \text { residue } \bmod p .
\end{array}\right.
$$

The Jacobi symbol $\left(\frac{m}{s}\right)$ is defined in terms of the Legendre symbol as follows. If $s=p_{1}^{e_{1}} p_{2}^{e_{2}} \ldots p_{g}^{e_{g}}$ is the factorization of the odd number $s$ into a product of primes, and $m$ is relatively prime to $s$, then [4]

$$
\left(\frac{m}{s}\right)=\left(\frac{m}{p_{1}}\right)^{e_{1}}\left(\frac{m}{p_{2}}\right)^{e_{2}} \cdots\left(\frac{m}{p_{g}}\right)^{e_{g}}
$$

We list some properties about the Legendre and Jacobi symbols that may be useful to us in the encrypting process. The proofs to most of these can be found in [4]. Assume that $a$ and $b$ are integers, and that $m$ and $n$ are any odd numbers greater than 1 .

1. $\left(\frac{a^{2}}{m}\right)=1$ for any integer $a$.

2. If $a \equiv b \bmod m,\left(\frac{a}{m}\right)=\left(\frac{b}{m}\right)$. 
3. $\left(\frac{a b}{m}\right)=\left(\frac{a}{m}\right)\left(\frac{b}{m}\right)$.

4. $\left(\frac{-1}{m}\right)=(-1)^{\frac{m-1}{2}}$.

5. $\left(\frac{2}{m}\right)=(-1)^{\frac{m^{2}-1}{8}}$

6. $\left(\frac{m}{n}\right) \frac{n}{m}=(-1)^{\left[\frac{m-1}{2}\right]\left[\frac{n-1}{2}\right]}$.

7. The Jacobi symbol $\left(\frac{m}{s}\right)= \pm 1$ for any $m$ coprime to $s$. If $\left(\frac{m}{s}\right)=1$, it does not follow that $m$ is a square $\bmod m$. For example, the units modulo 15 are 1 , $2,4,7,8,11,13$ and 14 , and their squares are 1 and 4 . So 2 is not a square modulo 15 , but $\left(\frac{2}{15}\right)=\left(\frac{2}{3}\right)\left(\frac{2}{5}\right)=(-1)(-1)=1[4]$.

Now that we have the general encrypting process we need to discuss certain 'size' issues of the numbers. If we did not want to use music, then we would not need to concern ourselves with the magnitude of the numbers. However, the numbers need to abide by a certain structure, that is to say, we need to bound the size of the entries of the message vector $\vec{m}$.

In absolute value, let $|\alpha|$ be the maximal entry of $C$ and $\vec{m}$. As usual, let $C$ be a $n \times n$ matrix and $\vec{m}$ be a $n \times 1$ vector. This means that the largest number we can have is $n \alpha^{2}$. If we restrict ourselves to have only four notes per bar in our 'composition', then this means that $n \alpha^{2} \leq 9999$. We get the upperbound of 9999 since this is the largest four-digit number. Since $\alpha \in \mathbb{Z}$, then in the case that $n=20$, 
$|\alpha|=22$. If $n=30$, then $\alpha=18$. So, as we increase $n$, the value of $\alpha$ decreases.

There exist at least two ways to resolve this issue. The first is to consider increasing the number of notes per bar from four to eight. This means that $\alpha \in \mathbb{Z}$ must satisfy $n \alpha^{2} \leq 99,999,999$. Thus, for $n=30,|\alpha|=1825$. In general, if we want $k$ notes per bar, then

$$
|\alpha| \leq\left\lfloor\sqrt{\frac{10^{k}-1}{n}}\right\rfloor .
$$

The second is to consider the use of sparse matrices. So, we not only have the permutation matrix $P$, but we make sure that $L$ and $U$ also have their non-zero entries equal to \pm 1 . Now, when we multiply $L$ and $U$, it may no longer be a sparse matrix. However, the fact that their non-zero entries are \pm 1 ensures that the largest entry in the matrix $L U$ can ever be is $n$, as $L$ and $U$ are of course $n \times n$ matrices. How many times can $n$ occur? The answer is once. In fact, the worse case scenario is the following:

$$
\pm\left(\begin{array}{ccccc}
1 & 0 & 0 & \ldots & 0 \\
1 & 1 & 0 & \ldots & 0 \\
\vdots & \vdots & \ddots & \ddots & \vdots \\
1 & 1 & \ldots & 1 & 0 \\
1 & 1 & \ldots & 1 & 1
\end{array}\right) \times( \pm)\left(\begin{array}{ccccc}
1 & 1 & 1 & \ldots & 1 \\
0 & 1 & 1 & \ldots & 1 \\
\vdots & \ddots & \ddots & \ddots & \vdots \\
0 & 0 & \ddots & 1 & 1 \\
0 & 0 & \ldots & 0 & 1
\end{array}\right)=
$$




$$
\pm\left(\begin{array}{ccccc}
1 & 1 & \ldots & 1 & 1 \\
1 & 2 & \ldots & 2 & 2 \\
\vdots & \vdots & \ldots & \vdots & \vdots \\
1 & 2 & \ldots & (n-1) & (n-1) \\
1 & 2 & \ldots & (n-1) & n
\end{array}\right) .
$$

The proof of this is a simple application of mathematical induction.

Proposition 4.2.1 Let $L$ and $U$ respectively be lower and upper triangular $n \times n$ matrices whose non-zero entries are either all +1 or -1 . Then their product is the $n \times n$ matrix in Equation (4.5). Moreover, the product is the worse case scenario.

Proof. In the case of $n=1$, the result is immediate. Let us consider the case when $n=2$. We have:

$$
\pm\left(\begin{array}{ll}
1 & 0 \\
1 & 1
\end{array}\right) \times( \pm)\left(\begin{array}{ll}
1 & 1 \\
0 & 1
\end{array}\right)= \pm\left(\begin{array}{ll}
1 & 1 \\
1 & 2
\end{array}\right) .
$$

We assume that result holds for $n=N-1$. Then, consider $n=N$. We take our $N \times N$ matrix and respectively form the block matrices for $L$ and $U$ :

$$
\left(\begin{array}{cc}
L_{N-1} & 0_{N-1} \\
\pm 1_{N-1}^{T} & \pm 1
\end{array}\right) \text { and }\left(\begin{array}{cc}
U_{N-1} & \pm 1_{N-1} \\
0_{N-1}^{T} & \pm 1
\end{array}\right)
$$


where $\pm 1_{N-1}$ and $0_{N-1}$ are $(N-1) \times 1$ vectors whose entries are respectively \pm 1 and 0. $L_{N-1}$ and $U_{N-1}$ are respectively the lower and upper triangular matrices whose non-zero entries are \pm 1 . Multiplying these matrices we obtain the following:

$$
\left(\begin{array}{cc}
L_{N-1} U_{N-1} & L_{N-1}\left( \pm 1_{N-1}\right) \\
\pm 1_{N-1}^{T} U_{N-1} & \pm 1_{N-1}^{T}\left( \pm 1_{N-1}\right) \pm 1
\end{array}\right) .
$$

1. For $L_{N-1} U_{N-1}$, we apply the induction hypothesis.

2. $L_{N-1}\left( \pm 1_{N-1}\right)=$

$$
\pm\left(\begin{array}{cccc}
1 & 0 & \cdots & 0 \\
1 & 1 & \ddots & 0 \\
\vdots & \ddots & \ddots & \vdots \\
1 & 1 & \cdots & 1
\end{array}\right) \times( \pm)\left(\begin{array}{c}
1 \\
1 \\
\vdots \\
1
\end{array}\right)= \pm\left(\begin{array}{c}
1 \\
2 \\
\vdots \\
(N-1)
\end{array}\right) .
$$

3. $\pm 1_{N-1}^{T} U_{N-1}=$

$$
\pm\left(\begin{array}{c}
1 \\
1 \\
\vdots \\
1
\end{array}\right)^{T} \times( \pm)\left(\begin{array}{cccc}
1 & 1 & \cdots & 1 \\
0 & 1 & \ddots & 1 \\
\vdots & \ddots & \ddots & \vdots \\
0 & 0 & \cdots & 1
\end{array}\right)= \pm\left(\begin{array}{c}
1 \\
2 \\
\vdots \\
(N-1)
\end{array}\right)^{T}
$$

4. $\pm 1_{N-1}^{T}\left( \pm 1_{N-1}\right) \pm 1= \pm N$.

Putting the four together, we obtain the first part of the desired result. We now show that this is indeed the worse case scenario. By 'worse case scenario' we mean 
to say the matrices $L$ and $U$ have the most non-zero terms. If we first consider the case when their entries are either zero or one, then multiplying them we obtain the following:

$$
\pm\left(\begin{array}{cccc}
1 & 0 & \ldots & 0 \\
\vdots & \ddots & \ddots & \vdots \\
\vdots & \ddots & \ddots & 0 \\
1 & \ldots & \ldots & 1
\end{array}\right) \times( \pm)\left(\begin{array}{cccc}
1 & \ldots & \ldots & 1 \\
0 & \ddots & \ddots & \vdots \\
\vdots & \ddots & \ddots & \vdots \\
0 & \ldots & 0 & 1
\end{array}\right)= \pm\left(\begin{array}{cccc}
1 & 1 & \ldots & 1 \\
1 & 2 & \ldots & 2 \\
\vdots & \vdots & \ddots & \vdots \\
1 & 2 & \ldots & n
\end{array}\right)
$$

It is clear that if instead of +1 we have -1 , the entries in the product are the same. Moreover, if exactly one of $L$ and $U$ has nonnegative entries and the other has nonpositive entries then the entries in the product are all negative. We note that in absolute value, the entries in the product $L U$ are at most those in Equation (4.6) for any matrices $L$ and $U$. Indeed, let $L U=A$ so that a typical $a_{i j}$ entry of $A$ is $a_{i j}=\sum_{k=1}^{n} l_{i k} u_{k j}$. Then, $\left|a_{i j}\right| \leq \min \{i, j\}$, i.e., $-\min \{i, j\} \leq a_{i j} \leq \min \{i, j\}$. This shows that the matrix in Equation (4.5) is indeed the worse case scenario.

Clearly, we observe that if $L$ and $U$ are $n \times n$ matrices, it may be possible that the maximal value of an entry in absolute value could be less than $n$. Thus, assuming that in absolute value the largest any entry can ever be in the product $P L U$ is $n$, then in the product $P L U \vec{m}$ the largest value the entries can have is

$$
\frac{n(n+1)}{2} \times|\alpha|,
$$


where $|\alpha|$ is the largest absolute value an entry of $\vec{m}$ can take. If we restrict ourselves in having only four notes per bar, this means that $\frac{n(n+1)}{2} \times|\alpha| \leq 9999$. Consider the case $n=30$, then as $\alpha \in \mathbb{Z}$, then $|\alpha|=21$. This value can only occur once as the permutation matrix $P$ permutes the rows.

The use of sparse matrices does not seem to have made a significant improvement. If we compare $|\alpha|$ when $n=30$ and we allow four notes per bar, we see that there has been an increase of 3 to $|\alpha|$. However, if we now allow eight notes per bar, then $|\alpha|$ goes from 1825 to 215053 , an increase of 213228 . Therefore, using sparse matrices is more efficient.

Example 4.2.3 Let us consider an example for $n=10$ to illustrate the encrypting process that we have established so far. Suppose that our message is numerically converted to:

$$
m=43214547914321610000012315798913231146133543268471
$$


and that

$$
C=P L U=\left(\begin{array}{ccccccccccc}
-1 & 1 & 0 & 0 & 0 & 1 & 0 & 0 & 0 & -1 \\
1 & 0 & -1 & 1 & 0 & -1 & 0 & -1 & 2 & 1 \\
0 & 1 & 0 & 1 & -1 & -1 & -1 & -1 & 2 & 1 \\
-1 & 1 & -1 & 1 & 1 & -1 & -1 & 1 & 1 & 2 \\
1 & 0 & 0 & 1 & -2 & 0 & -1 & -2 & 3 & 1 \\
0 & 0 & 0 & 0 & 0 & 1 & 0 & -2 & 0 & 1 \\
0 & -1 & 0 & 0 & 0 & 0 & 1 & 1 & -1 & -1 \\
1 & 0 & -1 & 0 & 1 & -2 & -1 & 1 & 1 & 2 \\
1 & -1 & 1 & -2 & 0 & 0 & -1 & 1 & 0 & 1 \\
0 & -1 & -1 & 2 & -1 & 0 & 2 & 0 & 1 & -2
\end{array}\right) .
$$

Next, we need to 'break' the number $m$ into vector components $m_{i}{ }^{5}$ to create a vector $\vec{m}$. Due to the use of sparse matrices and allowing eight notes per bar, we know that $\left|m_{i}\right| \leq 215053$. We separate $m$ into blocks of length five. We chose five because 215053 has length six and so we avoid possible problems when it will come time to convert the numbers into music. This will be made clear in an example later. In general if $\alpha$ has length $l_{\alpha}$, then we will break the number $m$ into blocks of size $l_{\alpha}-1$. In our case the length of $m$ is fifty so it is divisible by five. If this were

${ }^{5}$ Some confusion may arise with our notation. When speaking of vector components $m_{i}$, the index begins at one. If we are discussing about the digits of a number $m$, then the index begins at zero. 
not the case, then we would simply pad a sufficient number of zeroes to the left of the leading term. Let us say that after this process, our vector $\vec{m}$ is:

$\vec{m}=(-17486,23453,31641,-13231,-98975,13210,00001,61234,-19745,41234)^{T}$.

We note that we did not bother actually computing the Jacobi symbol of each vector component $m_{i}$. Instead, we randomly placed minus signs. Using the Jacobi symbol could potentially have some benefits and so we do not discard the possibility of it being used. Notice also that we have 'reversed' the order of the number $m$. That is, we fill the vector from bottom to top and write the digits right to left. This allows us to enter zeroes as 'leading' terms (left hand side of bottom number in $\vec{m}$ ) without altering the message. Upon decrypting, we need only to reverse this process. We further note that when we decrypt the seventh entry of $\vec{m}, 1$ would be returned. Since we know that the length of each vector component is five, we then know that $m_{7}=00001$. Moreover, we need only to take the absolute values of each entry. This being said, the product $P L U \vec{m}$ is:

$b=(12915,-135058,36496,7838,26763,-68024,16293,-50566,119631,-84792,)^{T}$.

At this moment, the mathematical encrypting part is finished. The next step is to send these numbers musically. We recall that we use the pentatonic scale to encode these numbers. So, we choose two scales, one being an octave higher than 
the other. By doing this, we make sure that the maximal difference in pitch is kept as small as possible. Then to each of the notes we assign a single digit. Because of the presence of minus signs, we need to encode these musically as well. Now, we have the following options.

1. Use some or all possible accents to represent minus signs.

i. An accent mark, $>$, that is placed above a note tells the musician to play the note loudly or hit hard depending on the instrument.

ii. A marcato accent, $\wedge$, that is placed above a note tells the musician to play the note very loudly or hit very hard.

iii. A stacatto mark, •, placed above a note means to play a short note or 'cut the sound.'

iv. A tenuto mark, - , placed above a note means to slightly lengthen the duration of the note.

2. Let the maximal absolute value that an entry of $\vec{m}$ can have be $\alpha$ and let the length of $\alpha$ be $l_{\alpha}$. In Example 4.2 .3 we mentioned that to avoid certain problems we broke the number $m$ into blocks of size $l_{\alpha}-1$. If we are to use $k$ notes per bar, then when multiplying $C \vec{m}=\vec{r}$, each entry of $\vec{r}$ will have length at most $k-1$. Thus, we still have space for an 'extra' note, i.e., the 
minus sign. We could simply assign some notes to represent the minus sign while the others would mean nothing.

i. We read the first note of each bar.

ii. If the first note corresponds to a minus sign, then we have a negative number and its digits are those that follow. If the first note does not correspond to a minus sign, then the note is 'ignored' numerically, i.e., we have a positive number and its digits are those that follow.

* For example, the even numbers represent a positive sign and the odd numbers represent a negative sign. Because we have five of each, we can have an appearance of 'randomness', i.e., it would be much harder to distinguish which numbers are even and which are odd.

3. The minus sign could represent a change in key. This idea however is not desirable. It may be the case that each vector component of $\vec{r}$ is negative and so we would have $n$ changes in key. Moreover, we would like to be able to change the $\mathrm{key}^{6}$ at our leisure without it (possibly) carrying any meaning, say to perhaps embellish the musical work.

Of the three options at our disposal, it would seem that only the first two should be considered. Now, the use of accents to represent minus signs is fine. However,

\footnotetext{
${ }^{6}$ We do not normally speak of 'keys' with pentatonicism, but rather of discreet 'pitch collections'.
} 
accents can be used in a much more powerful way and to use it to represent a minus sign would be a waste ${ }^{7}$. Thus, we use the second option.

We are getting a little closer in piecing all of this together to create our code. As mentioned previously, we use the pentatonic scale. To make the music pleasing, we employ two 'consecutive' pentatonic scales but in the bass clef, that is, if one is looking at a piano's keys, the pentatonic scales carrying the message are to the left of the middle $C$. Of course, we are not utilizing the bass clef exclusively. The treble clef is also used. However, the latter clef will only serve to accompany the bass notes to better harmonize. The treble notes we use are some pentatonic notes played in some random fashion. A point of concern about using the pentatonic scale is that overuse of it may result in a

vaguely Oriental-sounding tune or even something that sounds a little bit like the theme to an old cowboy movie [7].

However, we need not concern ourselves yet with this matter for now. At present, we only want to obtain a procedure that will allow us to convert numbers into music that is pleasing or at the very least, the numbers can generate music that 'makes sense'. So we have pentatonic notes in the same key as the pentatonic scales used in the bass clef, and the treble notes may be played in any order we want. The

\footnotetext{
${ }^{7}$ The use of accents could be used to hide some other information or to better encode the numbers. This will be discussed later.
} 
following tables indicate respectively which notes are to be used in the appropriate pentatonic scales according to the key. However, we first discuss about some of the notation used in the tables via the following definition.

Definition 4.2.6 We shall denote the pentatonic notes by $\nu$ the pentatonic notes being used by the bass clef. Moreover, the subscript $i$ indicates which note is being used, that is 0 for the first, 1 for the second, 2 for the third, 3 for the fifth and 4 for the sixth. Furthermore, to distinguish between octaves when we are using pentatonic notes, the notes of the higher octave shall have higher subscripts. Thus, the subscripts $5,6,7,8$ and 9 shall respectively represent the first, second, third, fifth and sixth of the higher octave.

Table 4.2.1

\begin{tabular}{|c||c|c|c|c|c|}
\hline \multicolumn{6}{|c|}{ Pentatonic 'Notes' } \\
\hline Key Signature & First & Second & Third & Fifth & Sixth \\
$\left(\nu_{0}, \nu_{5}\right)$ & $\left(\nu_{1} \nu_{6}\right)$ & $\left(\nu_{2} \nu_{7}\right)$ & $\left(\nu_{3} \nu_{8}\right)$ & $\left(\nu_{4} \nu_{9}\right)$ \\
\hline $\mathrm{C}$ & $\mathrm{C}$ & $\mathrm{D}$ & $\mathrm{E}$ & $\mathrm{G}$ & $\mathrm{A}$ \\
\hline $\mathrm{C} \sharp$ & $\mathrm{C} \sharp$ & $\mathrm{D} \sharp$ & $\mathrm{E} \sharp$ & $\mathrm{G} \sharp$ & $\mathrm{A} \sharp$ \\
\hline $\mathrm{Db}$ & $\mathrm{Db}$ & $\mathrm{Eb}$ & $\mathrm{F}$ & $\mathrm{Ab}$ & $\mathrm{Bb}$ \\
\hline $\mathrm{D}$ & $\mathrm{D}$ & $\mathrm{E}$ & $\mathrm{F} \sharp$ & $\mathrm{A}$ & $\mathrm{B}$ \\
\hline $\mathrm{Eb}$ & $\mathrm{Eb}$ & $\mathrm{F}$ & $\mathrm{G}$ & $\mathrm{Bb}$ & $\mathrm{C}$ \\
\hline
\end{tabular}


Table 4.2.1 (cont.)

\begin{tabular}{|c||c|c|c|c|c|}
\hline \multicolumn{7}{|c|}{ Pentatonic 'Notes' } \\
\hline Key Signature & First & Second & Third & Fifth & Sixth \\
& $\left(\nu_{0}, \nu_{5}\right)$ & $\left(\nu_{1} \nu_{6}\right)$ & $\left(\nu_{2} \nu_{7}\right)$ & $\left(\nu_{3} \nu_{8}\right)$ & $\left(\nu_{4} \nu_{9}\right)$ \\
\hline $\mathrm{E}$ & $\mathrm{E}$ & $\mathrm{F} \sharp$ & $\mathrm{G} \sharp$ & $\mathrm{B}$ & $\mathrm{C} \sharp$ \\
\hline $\mathrm{F}$ & $\mathrm{F}$ & $\mathrm{G}$ & $\mathrm{A}$ & $\mathrm{C}$ & $\mathrm{D}$ \\
\hline $\mathrm{F} \sharp$ & $\mathrm{F} \sharp$ & $\mathrm{G} \sharp$ & $\mathrm{A} \sharp$ & $\mathrm{CH}$ & $\mathrm{D} \sharp$ \\
\hline $\mathrm{Gb}$ & $\mathrm{Gb}$ & $\mathrm{Ab}$ & $\mathrm{Bb}$ & $\mathrm{Db}$ & $\mathrm{Eb}$ \\
\hline $\mathrm{G}$ & $\mathrm{G}$ & $\mathrm{A}$ & $\mathrm{B}$ & $\mathrm{D}$ & $\mathrm{E}$ \\
\hline $\mathrm{Ab}$ & $\mathrm{Ab}$ & $\mathrm{Bb}$ & $\mathrm{C}$ & $\mathrm{Eb}$ & $\mathrm{F}$ \\
\hline $\mathrm{A}$ & $\mathrm{A}$ & $\mathrm{B}$ & $\mathrm{CH}$ & $\mathrm{E}$ & $\mathrm{F} \sharp$ \\
\hline $\mathrm{Bb}$ & $\mathrm{Bb}$ & $\mathrm{C}$ & $\mathrm{D}$ & $\mathrm{F}$ & $\mathrm{G}$ \\
\hline $\mathrm{B} b$ & $\mathrm{~B}$ & $\mathrm{CH}$ & $\mathrm{D} \sharp$ & $\mathrm{F} \sharp$ & $\mathrm{G} \sharp$ \\
\hline $\mathrm{Cb}$ & $\mathrm{D} b$ & $\mathrm{~Eb}$ & $\mathrm{~Gb}$ & $\mathrm{Ab}$ \\
\hline
\end{tabular}

\subsection{Summary of Required Algorithms}

In reality there is but one algorithm. However, to make the presentation clearer, we have divided the algorithm into smaller and thus more manageable algorithms. We make the following assumptions, observing that this is a symmetric encryption system 
1. The sender(s) and receiver(s) have knowledge of the $n \times n$ sparse matrices $P, L$ and $U$, where $A=L U$ and $C=P A$. We have that $P$ is a permutation matrix (Definition 4.2.3) and the matrices $L$ and $U$ form the $L U$-factorization of $A$ (Definition 4.2.2). In our example, $n=32$.

2. The sender(s) and receiver $(\mathrm{s})$ have a table of symbols $\mathbb{E}$ similar to Table 3.1.1. Of course, the table that would be used would be complete. There would be a second table indicating which digit $0,1, \ldots, 9$ is assigned to which pentatonic note $\nu_{0}, \nu_{1}, \ldots, \nu_{9}$.

3. The sender(s) and receiver(s) would have knowledge of a finite set of primes $\mathbb{P}_{k}$. From this set of primes, a pre-determined order would be 'agreed' upon. (We had discussed this when reiterating the Gödel numbering process.)

4. The number of notes per bar will be $k$. In our example, $k$ will be sixteen.

5. Since the value of $n$ is known, the maximal entry $\alpha$ of the vector $\vec{m}$ is determined. Moreover, the length $l_{\alpha}$ of $\alpha$ is known.

Essentially, the only information kept private are $n, k$, a table similar to Table 3.1.1 and of course the matrices. With these assumptions in mind we now present the required algorithms. 


\section{Algorithm 4.3.1 - Encoding Algorithm (Phase 1)}

This algorithm converts the message into a number $m$. In addition, this number is then transformed into a vector $\vec{r}$.

1. Create the message number $m=p_{1}^{e_{1}} p_{2}^{e_{2}} \ldots p_{t}^{e_{t}}=a_{0}+10 a_{1}+10^{2} a_{2}+\cdots+10^{z} a_{z}$, where $p_{i} \in \mathbb{P}_{k}$ and $e_{i} \in \mathbb{E}$.

2. Set $l=l_{\alpha}-1$. If the length of $m$ is not divisible by $l$, then pad 'leading zeroes' to $m$ so that its length is then divisible by $l$, as discussed in Example 4.2.3.

3. Reverse the order of $m$ and separate $m$ into blocks of size $l$ as shown in Example 4.2.3. Each block is an entry of the message vector $\vec{m}$. Therefore, if $\vec{m}=$ $\left(m_{1}, m_{2}, \ldots, m_{n}\right)^{T}$, then $m_{1}=a_{0} a_{1} \ldots a_{l-1}, m_{2}=a_{l} a_{l+1} \ldots a_{2 l-1}$, etc.

4. We add minus signs to the entries $m_{i}$ of $\vec{m}$. This is done either by using the Legendre or Jacobi symbol (Definition 4.2.5), or by 'randomly' placing the minus signs.

5. Multiply $C \vec{m}=P L U \vec{m}=\vec{r}$.

\section{Algorithm 4.3.2 - Encoding Algorithm (Phase 2)}

This algorithm converts the vector $\vec{r}$ into music. We again emphasize that the intended receiver need not have to listen to the music. If 'Alice' sends a message to 'Bob', he knows that she will be sending some music in a 'special' format. Should 
the message be intercepted, without the proper software to decode, all that the enemy has is music. Since the music makes sense one may simply discard it and not realize that there is some hidden information. The music is only there to convince others that they have not intercepted a secret message.

1. Since we are using sixteenth notes, each vector entry of $\vec{r}$ has length at most 15. Moreover, some entries may be negative. To musically distinguish a positive number from a negative one, we play a particular note. For example, use the even numbers to represent a positive number and the odd numbers to represent a negative number. In other words, if the first note represents say the number 2 , we do not 'read' the note as the number 2 , but that the number is positive.

2. Each digit of the entries of $\vec{r}$ is then converted to its pentatonic note $\nu_{i}$.

\section{Algorithm 4.3.3 - Decryption Algorithm}

1. We receive $\vec{r}=P L U \vec{m}$. Multiply $P^{T} \vec{r}=y$. Set $U \vec{m}=\vec{x}$.

2. By 'forward' substitution, solve $L x=\vec{y}$ for $\vec{x}$.

3. By 'back' substitution, solve $U \vec{m}=\vec{x}$ for $\vec{m}$.

4. Compute $\left|m_{i}\right|$ for all $i$. 
The next three algorithms are also required in the encryption and decryption processes. Let $L=\left[l_{i j}\right], U=\left[u_{i j}\right], \vec{r}=\left[r_{i}\right], \vec{x}=\left[x_{i}\right]$ and $\vec{y}=\left[y_{i}\right]$. We note that since the matrix $C$ is invertible and $L$ and $U$ are sparse matrices, then $l_{i, i} \neq 0$ and $u_{i, i} \neq 0$ for all $i$.

\section{Algorithm 4.3.4 - Forward Substitution Algorithm}

for $i$ from 1 to $n$ do:

$$
x_{i}=\frac{y_{i}-\sum_{j=1}^{i-1} l_{i j} x_{j}}{l_{i i}} .
$$

\section{Algorithm 4.3.5 - Back Substitution Algorithm}

for $i$ from $n$ downto 1 do:

$$
m_{i}=\frac{x_{i}-\sum_{j=i+1}^{n} u_{i j} m_{j}}{u_{i i}} .
$$

The final algorithms are fast algorithms when exponentiating integers and multiplying matrices. The fast matrix multiplication is due to Strassen. All justifications (although not complex) to the algorithms can be found in [6]. We now present the algorithms as shown in [6]. 


\section{Algorithm 4.3.6 - Repeated Squaring Algorithm}

Input: $a \in R$, where $R$ is a ring with unity and $n \in \mathbb{N}_{>0}$.

Output: $a^{n} \in R$.

1. $\{$ Binary representation of $n\}$

write $n=2^{k}+n_{k-1} \cdot 2^{k-1}+\cdots+n_{1} \cdot 2+n_{0}$, with all $n_{i} \in\{0,1\}$.

$b_{k} \leftarrow a$

2. for $i=k-1$ downto 0 do

$$
\text { if } n_{i}=1 \text { then } b_{i} \leftarrow b_{i+1}^{2} a \text { else } b_{i} \leftarrow b_{i+1}^{2}
$$

\section{3. return $b_{0}$}

\section{Algorithm 4.3.7 - Strassen's Fast Matrix Multiplication}

Input: $A, B \in R^{n \times n}$, where $R$ is a ring and $n=2^{k}$ for some $k \in \mathbb{N}$.

Output: The product matrix $A B \in R^{n \times n}$.

1. if $n=1$ then let $A=(a), B=(b)$ for some $a, b \in R$ and return $(a b)$.

2. write $A=\left(\begin{array}{cc}A_{11} & A_{12} \\ A_{21} & A_{22}\end{array}\right), B=\left(\begin{array}{cc}B_{11} & B_{12} \\ B_{21} & B_{22}\end{array}\right)$, with all $A_{i j}, B_{i j} \in R^{\frac{n}{2} \times \frac{n}{2}}$.

3. $S_{1} \leftarrow A_{21}+A_{22}, \quad T_{1} \leftarrow B_{12}+B_{11}$ 


$$
\begin{array}{ll}
S_{2} \leftarrow S_{1}-A_{11}, & T_{2} \leftarrow B_{22}-T_{1} \\
S_{3} \leftarrow A_{11}-A_{21}, & T_{3} \leftarrow B_{22}-B_{12} \\
S_{4} \leftarrow A_{12}-S_{2}, & T_{4} \leftarrow T_{2}-B_{21}
\end{array}
$$

4. call the algorithm recursively to compute

$$
\begin{array}{ll}
P_{1}=A_{11} B_{11}, & P_{5}=S_{1} T_{1}, \\
P_{2}=A_{12}-B_{21}, & P_{6}=S_{2} T_{2} \\
P_{3}=S_{4}-B_{22}, & P_{7}=S_{3} T_{3} . \\
P_{4}=A_{22}-T_{4}, &
\end{array}
$$

5. $U_{1} \leftarrow P_{1}+P_{2}, \quad U_{5} \leftarrow U_{4}+P_{3}$,

$$
U_{2} \leftarrow P_{1}-P_{6}, \quad U_{6} \leftarrow U_{3}-P_{4},
$$$$
U_{3} \leftarrow U_{2}+P_{7}, \quad U_{7} \leftarrow U_{3}+P_{5} .
$$$$
U_{4} \leftarrow U_{2}+P_{5},
$$

6. return $\left(\begin{array}{cc}U_{1} & U_{5} \\ U_{6} & U_{7}\end{array}\right)$.

\subsection{Remarks and Examples}

We provide an example to help illustrate the encryption and coding processes. However, before we proceed with the example, we require some needed elements. 
1. A table of permissible symbols similar to Table 4.4.1, on page 63. From [14], the 95 basic symbols are numbered from 32 to 126 . To create our table, we subtract 31 from each to obtain the new table. Noting that the lower case letters are represented by some of the largest numbers, we reassign the ninetyfive numbers to make the example a little more manageable.

2. A set of primes and the order in which they occur must be determined. For example, we may decide to use the prime three to denote the tenth symbol, i.e., $p_{10}=3$. In our example however, we do not use the primes 2 and 5 to remind the reader that certain primes need not be included in the set. However, for simplicity, if $i<j$, then $p_{i}<p_{j}$.

3. Matrices $P, L$ and $U$, such that $C=P L U$. Our matrices will be randomly generated. In practice, this can be done by various means. 
Table 4.4.1

\begin{tabular}{|c|c|c|c|c|c|c|c|}
\hline Number & Symbol & Number & Symbol & Number & Symbol & Number & Symbol \\
\hline 1 & space & 2 & a & 3 & $\mathrm{~b}$ & 4 & $\mathrm{c}$ \\
\hline 5 & d & 6 & $\mathrm{e}$ & 7 & f & 8 & $\mathrm{~g}$ \\
\hline 9 & $\mathrm{~h}$ & 10 & $\mathrm{i}$ & 11 & $\mathrm{j}$ & 12 & $\mathrm{k}$ \\
\hline 13 & 1 & 14 & $\mathrm{~m}$ & 15 & . & 16 & $\mathrm{n}$ \\
\hline 17 & o & 18 & $\mathrm{p}$ & 19 & $q$ & 20 & $\mathrm{r}$ \\
\hline 21 & $\mathrm{~S}$ & 22 & $t$ & 23 & $\mathrm{u}$ & 24 & $\mathrm{v}$ \\
\hline 25 & $\mathrm{~W}$ & 26 & $\mathrm{x}$ & 27 & $\mathrm{y}$ & 28 & $\mathrm{z}$ \\
\hline 29 & 0 & 30 & 1 & 31 & 2 & 32 & 3 \\
\hline 33 & 4 & 34 & A & 35 & B & 36 & $\mathrm{C}$ \\
\hline 37 & D & 38 & $\mathrm{E}$ & 39 & $\mathrm{~F}$ & 40 & $\mathrm{G}$ \\
\hline 41 & $\mathrm{H}$ & 42 & I & 43 & $J$ & 44 & K \\
\hline 45 & $\mathrm{~L}$ & 46 & M & 47 & $\mathrm{~N}$ & 48 & $\mathrm{O}$ \\
\hline 49 & $\mathrm{P}$ & 50 & $\mathrm{Q}$ & 51 & $\mathrm{R}$ & 52 & $\mathrm{~S}$ \\
\hline 53 & $\mathrm{~T}$ & 54 & $U$ & 55 & $\mathrm{~V}$ & 56 & W \\
\hline 57 & $\mathrm{X}$ & 58 & $\mathrm{Y}$ & 59 & $\mathrm{Z}$ & 60 & , \\
\hline 61 & 1 & 62 & ] & 63 & {[} & 64 & - \\
\hline 65 & 6 & 66 & $?$ & 67 & 5 & 68 & 6 \\
\hline
\end{tabular}


Table 4.4.1 (cont.)

\begin{tabular}{|c|c||c|c||c|c||c|c|}
\hline Number & Symbol & Number & Symbol & Number & Symbol & Number & Symbol \\
\hline 69 & 7 & 70 & 8 & 71 & 9 & 72 & $\wedge$ \\
\hline 73 & $@$ & 74 & $"$ & 75 &, & 76 & ( \\
\hline 77 & ) & 78 & + & 79 & - & 80 & $=$ \\
\hline 81 & $:$ & 82 & $;$ & 83 & $>$ & 84 & $<$ \\
\hline 85 & $!$ & 86 & $/$ & 87 & $*$ & 88 & $\&$ \\
\hline 89 & $\%$ & 90 & $\$$ & 91 & $\#$ & 92 & \{ \\
\hline 93 & 1 & 94 & \} & 95 & $\sim$ & & \\
\hline
\end{tabular}

Example 4.4.1 Let us send the message: This is a test. We note that all of the large multiplications were done using a 'Big Integer Calculator' at [13] and all large matrix multiplications were done using a 'Large Matrix Multiplier' [17]. Referring to Table 4.4.1, we require in order the following exponents for our primes: $53,9,10$, $21,1,9,21,1,2,1,22,6,21,22$ and 15 . We need the 23 primes: $3,7,11,13,17$, $19,23,29,31,37,41,43,47,53,59$ and 61 . That is to say,

'This': $3^{53} \times 7^{9} \times 11^{10} \times 13^{21} \times 17^{1}=$ 85210876320587099248078349263350630568659329293851361621641505494681. 'is': $19^{9} \times 23^{21} \times 29^{1}=$ 369372843620323451868956302128443557872193. 
'a': $31^{2} \times 37^{1}=35557$.

'test.': $41^{22} \times 43^{6} \times 47^{21} \times 53^{22} \times 59^{15}=$

78155564422120416681903140133447841879115055504339428766030765882489

43744848249698254459566275954251829146744648340867615770380277101804

115707613

Thus, 'This $\times$ is $\times a \times$ test.' becomes $m=$

87467156890648548204170810009368647085408793689800186889960069523438

60220331106004917473244829766318397981573547412292766371454782292931

14278108701195251591496647739594335860672763614314122551992402387193

0471153195562226637615450119429808459587228548361330953,

and we note that $m$ has length 259. Since we can have at most sixteen notes per bar, this means that, in absolute value, the maximal entry $|\alpha|$ that the vector $\vec{m}$ can have is:

$$
|\alpha| \times \frac{n(n+1)}{2} \leq 10^{16-1}-1 \Rightarrow|\alpha|=1893939393939
$$

for $n=32$. We chose $n=32$ because in Algorithm 4.3.7, $n$ must be a power of 2 . (Actually, one can always pad zeroes to the next power of 2.) Since the length of $\alpha$ is $l_{\alpha}=13$, then every entry of $\vec{m}$ should have length at most twelve. We then calculate $12 \times 32=384=259+125$ and so we add 125 leading zeroes to the number $m$ as discussed in Example 4.2.3. Recall also that we reverse the order of $m$ when 
creating $\vec{m}$ and add negative signs in a random fashion to obtain:

$\vec{m}=(-359033163845,-822785954808,924911054516,-736622265591$, - 351174039178, 320429915522, 141341636727, -606853349593, 774669419515, 259110780187, 241139292287, -454173667292, -214745375189, 793813667928, - 442374719400, -601133022068, 343259600699, 886810089863, -978045807468, - 639000180714,028458460986, 517647800000,000000000000, 000000000000, $000000000000,000000000000,000000000000,000000000000,000000000000$, $000000000000,000000000000,000000000000)^{T}$.

Let $P, L$ and $U$ be $32 \times 32$ matrices and $C=P L U$. To make the matrices fit on this page, we shall use block matrices. So, for a $32 \times 32$ matrix $A$, we have that

$$
A=\left(\begin{array}{ll}
A_{1} & A_{2} \\
A_{3} & A_{4}
\end{array}\right),
$$


where the $A_{i}$ are $16 \times 16$ block-matrices. We obtain the following:

$$
L_{1}=\left(\begin{array}{rrrrrrrrrrrrrrrr}
1 & 0 & 0 & 0 & 0 & 0 & 0 & 0 & 0 & 0 & 0 & 0 & 0 & 0 & 0 & 0 \\
-1 & 1 & 0 & 0 & 0 & 0 & 0 & 0 & 0 & 0 & 0 & 0 & 0 & 0 & 0 & 0 \\
1 & 0 & 1 & 0 & 0 & 0 & 0 & 0 & 0 & 0 & 0 & 0 & 0 & 0 & 0 & 0 \\
0 & 0 & 0 & -1 & 0 & 0 & 0 & 0 & 0 & 0 & 0 & 0 & 0 & 0 & 0 & 0 \\
1 & 0 & 0 & 0 & -1 & 0 & 0 & 0 & 0 & 0 & 0 & 0 & 0 & 0 & 0 & 0 \\
0 & 0 & 1 & 0 & 0 & -1 & 0 & 0 & 0 & 0 & 0 & 0 & 0 & 0 & 0 & 0 \\
-1 & 0 & 0 & 0 & 0 & 0 & 1 & 0 & 0 & 0 & 0 & 0 & 0 & 0 & 0 & 0 \\
1 & 0 & 1 & 0 & 0 & 0 & 0 & 1 & 0 & 0 & 0 & 0 & 0 & 0 & 0 & 0 \\
0 & 0 & 1 & 0 & -1 & 0 & 1 & 0 & 1 & 0 & 0 & 0 & 0 & 0 & 0 & 0 \\
-1 & 0 & 0 & 1 & 0 & 1 & 0 & -1 & 0 & 1 & 0 & 0 & 0 & 0 & 0 & 0 \\
1 & 0 & 0 & 1 & 0 & -1 & 0 & 0 & 1 & 0 & 1 & 0 & 0 & 0 & 0 & 0 \\
0 & 0 & 1 & 0 & 1 & 0 & 0 & 0 & 0 & -1 & 0 & 1 & 0 & 0 & 0 & 0 \\
1 & 0 & 0 & 1 & 0 & 0 & 1 & 0 & 0 & -1 & 0 & 0 & 1 & 0 & 0 & 0 \\
-1 & 0 & 0 & 1 & 0 & -1 & 0 & 0 & 0 & 0 & 1 & 0 & 0 & -1 & 0 & 0 \\
1 & 0 & 1 & 1 & -1 & 0 & 0 & 1 & 0 & -1 & 0 & 1 & 0 & -1 & 1 & 0 \\
0 & 0 & 1 & 0 & 1 & 0 & -1 & 0 & 0 & 0 & -1 & 0 & 0 & 1 & 0 & -1
\end{array}\right),
$$


$L_{2}$ is a $16 \times 16$ zero-matrix,

$$
\left.\begin{array}{rrrrrrrrrrrrrrrr}
-1 & 0 & 0 & 1 & 0 & 0 & 0 & -1 & 0 & 0 & 0 & 1 & 0 & 1 & 0 & -1 \\
1 & 0 & 0 & 0 & 0 & -1 & 0 & 0 & 0 & 1 & 0 & 0 & 0 & 0 & 1 & 0 \\
0 & 0 & 0 & -1 & 0 & 0 & 0 & 1 & 0 & 0 & 1 & 0 & 0 & -1 & 0 & 0 \\
1 & 0 & 0 & 0 & -1 & 0 & 0 & 1 & 0 & 0 & 0 & -1 & 0 & 0 & 0 & -1 \\
1 & 0 & 0 & -1 & 0 & 0 & -1 & 0 & 1 & 0 & -1 & 0 & 0 & 0 & 0 & 0 \\
0 & 0 & 0 & 1 & 0 & 0 & 0 & -1 & 0 & 0 & 1 & -1 & 0 & 0 & 1 & 0 \\
1 & 0 & -1 & 0 & 0 & 1 & 0 & 0 & -1 & 0 & 0 & 0 & 1 & 0 & 0 & 1 \\
0 & 0 & 0 & -1 & 0 & 0 & 1 & 0 & 0 & 0 & 0 & 1 & 0 & 1 & 0 & 0 \\
1 & 0 & 0 & 1 & 0 & -1 & 0 & 0 & 0 & 1 & 0 & -1 & 0 & 0 & 1 & 0 \\
-1 & 0 & 0 & 1 & -1 & 0 & 1 & 0 & 0 & 0 & 1 & 0 & -1 & -1 & 1 & 0 \\
1 & 0 & -1 & 0 & 0 & 1 & 0 & 0 & 1 & 0 & 0 & -1 & 0 & 0 & 1 & 0 \\
0 & 0 & -1 & 0 & 0 & 1 & 0 & -1 & 0 & 0 & 1 & 0 & 1 & 0 & 0 & 1 \\
1 & 0 & 0 & -1 & 0 & 0 & 0 & 0 & -1 & 0 & 0 & 0 & 1 & 0 & 0 & 1 \\
-1 & 0 & 0 & 1 & 0 & 0 & 0 & 0 & 0 & 0 & 0 & -1 & 0 & 1 & 0 & -1 \\
0 & 0 & 0 & 0 & -1 & 0 & 1 & 0 & -1 & 0 & 1 & 0 & 0 & 0 & -1 & 0 \\
1 & 0 & 0 & 0 & -1 & 0 & 0 & 1 & 0 & 0 & 0 & -1 & 0 & 1 & 0
\end{array}\right)
$$


and

$$
L_{4}=\left(\begin{array}{cccccccccccccccc}
1 & 0 & 0 & 0 & 0 & 0 & 0 & 0 & 0 & 0 & 0 & 0 & 0 & 0 & 0 & 0 \\
0 & -1 & 0 & 0 & 0 & 0 & 0 & 0 & 0 & 0 & 0 & 0 & 0 & 0 & 0 & 0 \\
0 & 0 & 1 & 0 & 0 & 0 & 0 & 0 & 0 & 0 & 0 & 0 & 0 & 0 & 0 & 0 \\
0 & 0 & 0 & 1 & 0 & 0 & 0 & 0 & 0 & 0 & 0 & 0 & 0 & 0 & 0 & 0 \\
0 & 1 & 0 & 0 & 1 & 0 & 0 & 0 & 0 & 0 & 0 & 0 & 0 & 0 & 0 & 0 \\
0 & 0 & 1 & -1 & 0 & 1 & 0 & 0 & 0 & 0 & 0 & 0 & 0 & 0 & 0 & 0 \\
0 & 0 & 0 & 0 & 1 & 0 & -1 & 0 & 0 & 0 & 0 & 0 & 0 & 0 & 0 & 0 \\
0 & 0 & 1 & 0 & 0 & 1 & 0 & 1 & 0 & 0 & 0 & 0 & 0 & 0 & 0 & 0 \\
0 & 1 & 0 & 1 & 0 & 0 & 0 & 0 & -1 & 0 & 0 & 0 & 0 & 0 & 0 & 0 \\
1 & 0 & 0 & -1 & 0 & 0 & 0 & 1 & 0 & 0 & 1 & 0 & 0 & 0 & 0 & 0 \\
0 & 1 & 0 & 0 & 0 & 0 & -1 & 0 & 0 & 1 & 0 & 1 & 0 & 0 & 0 & 0 \\
0 & -1 & 0 & 1 & 0 & 0 & 0 & -1 & 0 & 0 & 1 & 0 & 1 & 0 & 0 & 0 \\
0 & 1 & 0 & 0 & 0 & 1 & 0 & 0 & 1 & 0 & 0 & -1 & 0 & 1 & 0 & 0 \\
1 & 0 & -1 & 0 & 0 & 0 & -1 & 0 & 0 & 1 & 0 & 0 & 1 & 0 & -1 & 0 \\
0 & 0 & 0 & -1 & 0 & 1 & 0 & 1 & 0 & 0 & -1 & 0 & 0 & 1 & 0 & -1
\end{array}\right) .
$$


For $U$ we have

$$
U_{1}=\left(\begin{array}{cccccccccccccccc}
1 & 0 & 1 & 0 & 0 & -1 & 0 & 0 & 0 & 1 & 0 & 0 & 0 & 0 & -1 & 0 \\
0 & 1 & 0 & 0 & 1 & 0 & 0 & -1 & 0 & 0 & 0 & 1 & 0 & 1 & 0 & 0 \\
0 & 0 & 1 & 0 & 0 & 1 & 0 & 0 & 1 & 0 & 0 & 0 & 0 & 0 & -1 & 0 \\
0 & 0 & 0 & -1 & 0 & 0 & 0 & 1 & 0 & 0 & 0 & 1 & 0 & 0 & 0 & 1 \\
0 & 0 & 0 & 0 & 1 & 0 & 0 & 0 & 1 & 0 & 0 & 0 & 1 & 0 & 0 & 0 \\
0 & 0 & 0 & 0 & 0 & 1 & 0 & 0 & 0 & 1 & -1 & 0 & 1 & 0 & 0 & 1 \\
0 & 0 & 0 & 0 & 0 & 0 & -1 & 0 & 0 & 0 & 1 & 0 & 0 & 1 & 0 & 0 \\
0 & 0 & 0 & 0 & 0 & 0 & 0 & 1 & 1 & 0 & 0 & 1 & 0 & -1 & 1 & 0 \\
0 & 0 & 0 & 0 & 0 & 0 & 0 & 0 & 1 & 0 & 0 & 0 & 0 & 0 & 0 & -1 \\
0 & 0 & 0 & 0 & 0 & 0 & 0 & 0 & 0 & -1 & 0 & 0 & 1 & 0 & 0 & 0 \\
0 & 0 & 0 & 0 & 0 & 0 & 0 & 0 & 0 & 0 & 1 & 0 & 0 & 0 & 1 & 0 \\
0 & 0 & 0 & 0 & 0 & 0 & 0 & 0 & 0 & 0 & 0 & -1 & 0 & 1 & 0 & 0 \\
0 & 0 & 0 & 0 & 0 & 0 & 0 & 0 & 0 & 0 & 0 & 0 & 1 & 0 & 0 & 0 \\
0 & 0 & 0 & 0 & 0 & 0 & 0 & 0 & 0 & 0 & 0 & 0 & 0 & -1 & 0 & 1 \\
0 & 0 & 0 & 0 & 0 & 0 & 0 & 0 & 0 & 0 & 0 & 0 & 0 & 0 & 0 & 1
\end{array}\right),
$$




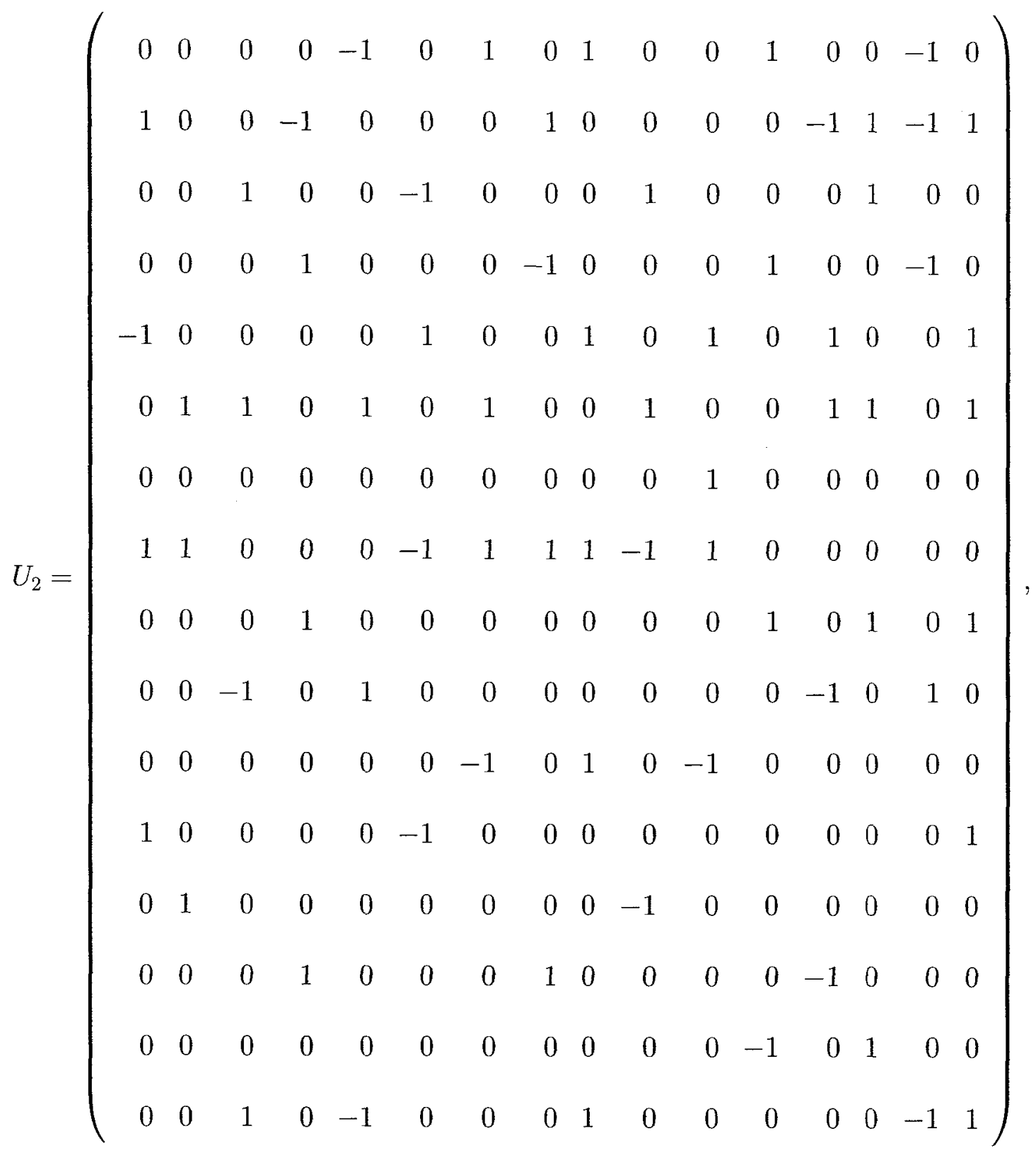


$U_{3}$ is a $16 \times 16$ zero-matrix and

$$
U_{4}=\left(\begin{array}{cccccccccccccccc}
-1 & 0 & 0 & 0 & 0 & 1 & 0 & 0 & 0 & -1 & 1 & 0 & -1 & 0 & 0 & 0 \\
0 & -1 & 0 & 1 & 0 & 0 & 0 & -1 & 0 & 0 & 0 & 0 & 0 & 0 & 0 & 0 \\
0 & 0 & 1 & 0 & 0 & 0 & -1 & 0 & 0 & 0 & 0 & 1 & 0 & 0 & -1 & 0 \\
0 & 0 & 0 & 1 & 0 & 1 & 0 & 0 & 0 & -1 & 0 & 0 & 0 & 1 & 0 & 0 \\
0 & 0 & 0 & 0 & -1 & 0 & 0 & 0 & 0 & 0 & 1 & 0 & 0 & 0 & -1 & 0 \\
0 & 0 & 0 & 0 & 0 & 1 & 0 & 0 & -1 & 0 & 0 & 0 & 1 & 0 & 0 & -1 \\
0 & 0 & 0 & 0 & 0 & 0 & 1 & 0 & 0 & 0 & 0 & 1 & 0 & -1 & 0 & 0 \\
0 & 0 & 0 & 0 & 0 & 0 & 0 & -1 & 0 & 1 & 0 & 0 & 0 & 0 & -1 & 0 \\
0 & 0 & 0 & 0 & 0 & 0 & 0 & 0 & -1 & 0 & 0 & 0 & 1 & 0 & 0 & 0 \\
0 & 0 & 0 & 0 & 0 & 0 & 0 & 0 & 0 & 1 & 0 & 0 & 0 & -1 & 0 & 0 \\
0 & 0 & 0 & 0 & 0 & 0 & 0 & 0 & 0 & 0 & 1 & 0 & 0 & 0 & 0 & 1 \\
0 & 0 & 0 & 0 & 0 & 0 & 0 & 0 & 0 & 0 & 0 & 1 & 0 & 0 & -1 & 0 \\
0 & 0 & 0 & 0 & 0 & 0 & 0 & 0 & 0 & 0 & 0 & 0 & -1 & 0 & 0 & 0 \\
0 & 0 & 0 & 0 & 0 & 0 & 0 & 0 & 0 & 0 & 0 & 0 & 0 & 1 & 0 & 0 \\
0 & 0 & 0 & 0 & 0 & 0 & 0 & 0 & 0 & 0 & 0 & 0 & -1 & 0 \\
0 & 0 & 0 & 0 & 0 & 0 & 0 & 0 & 0 & 0 & 1
\end{array}\right)
$$


Finally, for $P$ we have

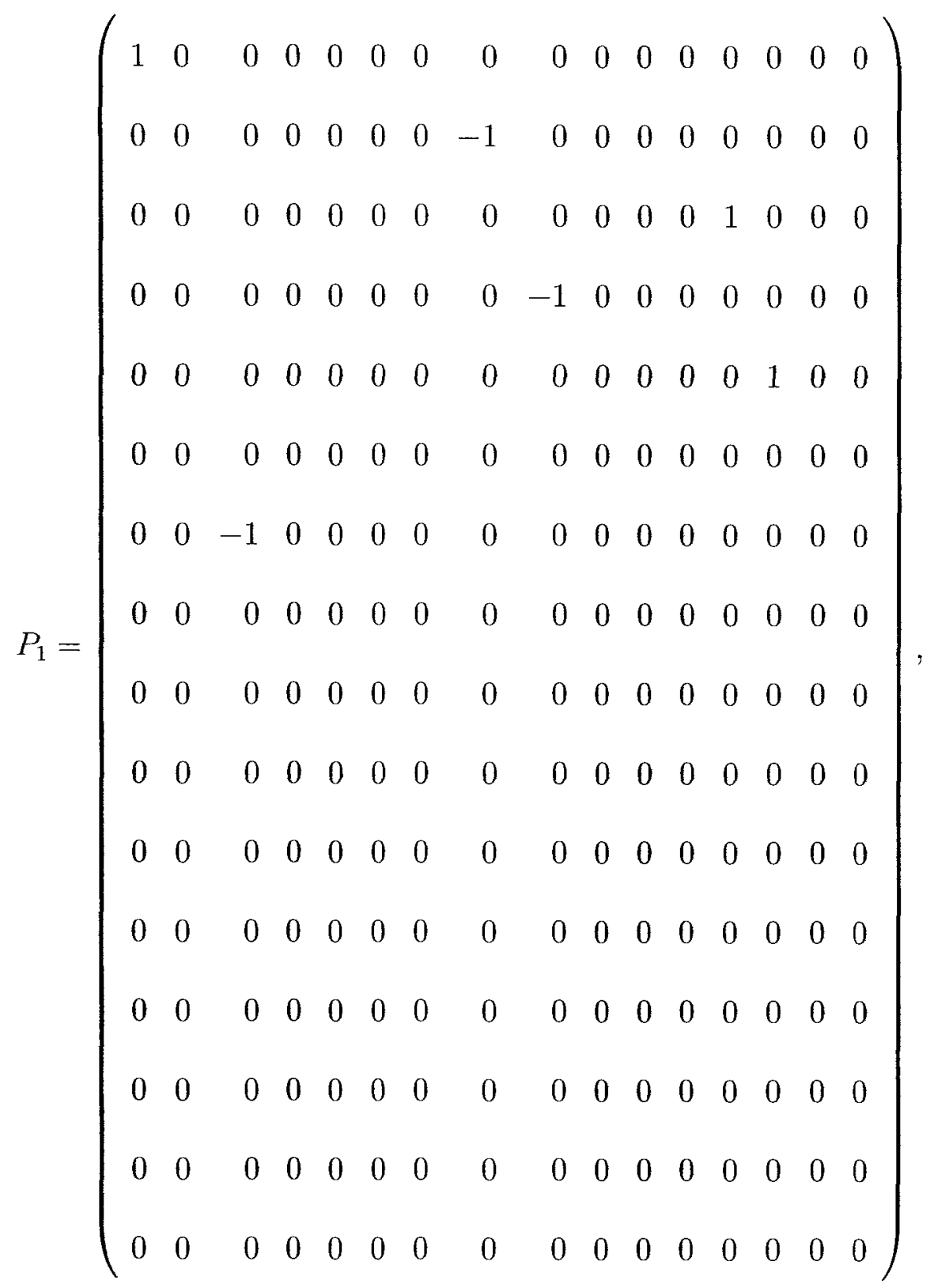




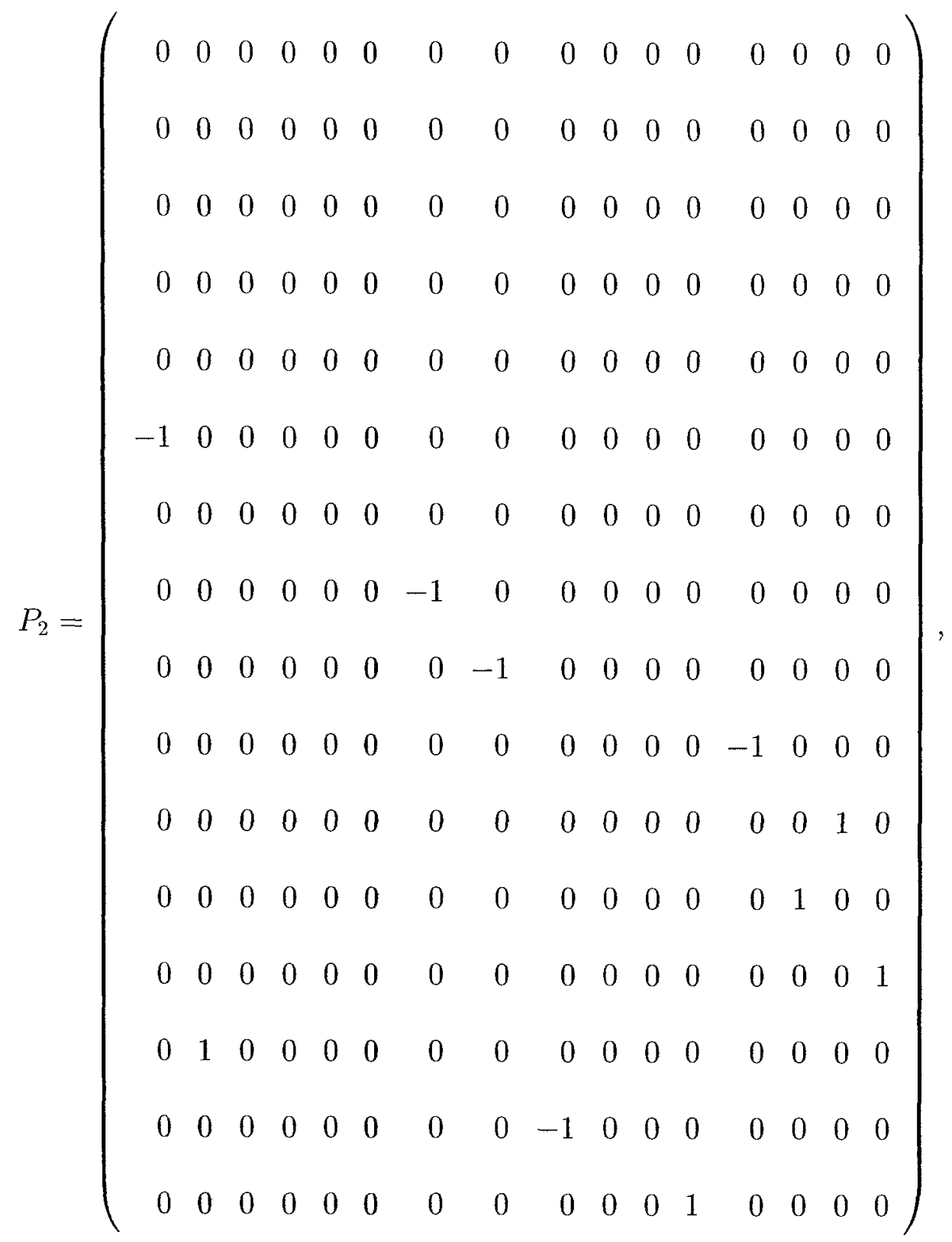




$$
P_{3}=\left(\begin{array}{cccccccccccccccc}
0 & 0 & 0 & 0 & 0 & 0 & 0 & 0 & 0 & 0 & 0 & 0 & 0 & 0 & 0 & 0 \\
0 & -1 & 0 & 0 & 0 & 0 & 0 & 0 & 0 & 0 & 0 & 0 & 0 & 0 & 0 & 0 \\
0 & 0 & 0 & 0 & 0 & 0 & 0 & 0 & 0 & 0 & 0 & 0 & 0 & 0 & 0 & 0 \\
0 & 0 & 0 & 0 & 0 & 0 & 0 & 0 & 0 & 0 & 0 & 0 & 0 & 0 & 0 & 0 \\
0 & 0 & 0 & 0 & 0 & 0 & 0 & 0 & 0 & 0 & 0 & 0 & 0 & 0 & 0 & 0 \\
0 & 0 & 0 & 0 & 0 & 0 & 0 & 0 & 0 & 0 & 0 & 0 & 0 & 0 & 0 & 0 \\
0 & 0 & 0 & -1 & 0 & 0 & 0 & 0 & 0 & 0 & 0 & 0 & 0 & 0 & 0 & 0 \\
0 & 0 & 0 & 0 & 0 & 0 & 0 & 0 & 0 & 0 & 0 & 0 & 0 & 0 & 0 & 0 \\
0 & 0 & 0 & 0 & 0 & 0 & -1 & 0 & 0 & 0 & 0 & 0 & 0 & 0 & 0 & 0 \\
0 & 0 & 0 & 0 & 0 & 0 & 0 & 0 & 0 & 0 & 0 & 0 & 0 & 0 & 1 & 0 \\
0 & 0 & 0 & 0 & 0 & 0 & 0 & 0 & 0 & 0 & 0 & 0 & 0 & 0 & 0 & 1 \\
0 & 0 & 0 & 0 & 0 & 0 & 0 & 0 & 0 & 0 & 1 & 0 & 0 & 0 & 0 & 0 \\
0 & 0 & 0 & 0 & 0 & 1 & 0 & 0 & 0 & 0 & 0 & 0 & 0 & 0 & 0 & 0 \\
0 & 0 & 0 & 0 & 0 & 0 & 0 & 1 & 0 & 0 & 0 & 0 & 0 & 0 \\
0 & 0 & 0 & 0 & 0 & 0 & 0 & 0 & 0 & 0 & 0 & 0 & 0 & 0
\end{array}\right)
$$


and

$$
P_{4}=\left(\begin{array}{cccccccccccccccc}
0 & 0 & 1 & 0 & 0 & 0 & 0 & 0 & 0 & 0 & 0 & 0 & 0 & 0 & 0 & 0 \\
0 & 0 & 0 & 0 & 0 & 0 & 0 & 0 & 0 & 0 & 0 & 0 & 0 & 0 & 0 & 0 \\
0 & 0 & 0 & 0 & 0 & 0 & 0 & 0 & 0 & 0 & 1 & 0 & 0 & 0 & 0 & 0 \\
0 & 0 & 0 & 0 & 0 & 0 & 0 & 0 & 0 & -1 & 0 & 0 & 0 & 0 & 0 & 0 \\
0 & 0 & 0 & 0 & 0 & -1 & 0 & 0 & 0 & 0 & 0 & 0 & 0 & 0 & 0 & 0 \\
0 & 0 & 0 & 0 & 1 & 0 & 0 & 0 & 0 & 0 & 0 & 0 & 0 & 0 & 0 & 0 \\
0 & 0 & 0 & 0 & 0 & 0 & 0 & 0 & 0 & 0 & 0 & 0 & 0 & 0 & 0 & 0 \\
0 & 0 & 0 & -1 & 0 & 0 & 0 & 0 & 0 & 0 & 0 & 0 & 0 & 0 & 0 & 0 \\
0 & 0 & 0 & 0 & 0 & 0 & 0 & 0 & 0 & 0 & 0 & 0 & 0 & 0 & 0 & 0 \\
0 & 0 & 0 & 0 & 0 & 0 & 0 & 0 & 0 & 0 & 0 & 0 & 0 & 0 & 0 & 0 \\
0 & 0 & 0 & 0 & 0 & 0 & 0 & 0 & 0 & 0 & 0 & 0 & 0 & 0 & 0 & 0 \\
0 & 0 & 0 & 0 & 0 & 0 & 0 & 0 & 0 & 0 & 0 & 0 & 0 & 0 & 0 & 0 \\
0 & 0 & 0 & 0 & 0 & 0 & 0 & 0 & 0 & 0 & 0 & 0 & 0 & 0 & 0 & 0 \\
0 & 0 & 0 & 0 & 0 & 0 & 0 & 0 & 0 & 0 & 0 & 0 & 0 & 0 & 0 & 0 \\
0 & 0 & 0 & 0 & 0 & 0 & 0 & 0 & 0 & 0 & 0 & 0 & 0 & 0 \\
0 & 0 & 0 & 0 & 0 & 0 & 0 & 0 & 0 & 0 & 0 & 0 & 0
\end{array}\right)
$$

Next, we calculate $C=P L U$ using Algorithm 4.3.7 twice, and then we compute $C \vec{m}=\vec{r}=$

(918475013750, - 1075042421099, 386964984758, -2213966881393, -110047273775, 
$851211118658,-1885166515235,2289304035892,-1211791734606,-2215096020972$, 282494718426, -4991084154510, 767891683013,3959562367259,-1200072896550, - 3359439931341, 1609079496069, 163681876094, - 187152313824, 13776800871041, $1925919330326,973170600757,-1564537954076,-137898489054,-1890780728775$, 24863690262, 231144229371, 429758143884, 1506945751939, 2144638875525, $-1680495011066,-535336809301)^{T}$.

The next step is converting $\vec{r}$ into music. To achieve this we require the following table, recalling Definition 4.2.6 and Table 4.2.1.

\section{Table 4.4 .2}

\begin{tabular}{|c|c|c|c|c||c|c|c|c|c|}
\hline$\nu_{0}$ & $\nu_{1}$ & $\nu_{2}$ & $\nu_{3}$ & $\nu_{4}$ & $\nu_{5}$ & $\nu_{6}$ & $\nu_{7}$ & $\nu_{8}$ & $\nu_{9}$ \\
\hline 7 & 1 & 6 & 3 & 0 & 8 & 9 & 2 & 4 & 5 \\
\hline- & - & + & - & + & + & - & + & + & - \\
\hline
\end{tabular}

All that remains is to generate a 'random sequence' of pentatonic notes to accompany the bass notes. The decryption process is straightforward. We perform the following calculations in order.

1. $P^{T} \vec{r}=\vec{y}$.

2. From $L \vec{x}=\vec{y}$, we solve for $\vec{x}$ by using Algorithm 4.3.4.

3. We solve for $\vec{m}$ from $U \vec{m}=\vec{x}$ by using Algorithm 4.3.5. 
4. We take the absolute values of each entry of $\vec{m}$. For sake of brevity, we simply denote them as $m_{1}, m_{2}, \ldots, m_{n}$. In our example, $n=32$.

5. We 'flip' each $m_{i}$, so that if $m_{i}$ has decimal expansion $a_{i_{k}} a_{i_{k-1}} \ldots a_{i_{0}}$, then by flipping we mean that $m_{i} \mapsto m_{i}^{\prime}$ where $m_{i}^{\prime}$ has decimal expansion $a_{i_{0}} a_{i_{1}} \ldots a_{i_{k}}$. Should $a_{i_{0}}=0$, we do not remove it. Thus, the number $m$, in decimal notation, is equal to $m_{n}^{\prime} m_{n-1}^{\prime} \ldots m_{0}^{\prime}$.

6. After removing (if any) leading zeroes, we simply factorize. Since we know which primes were used this task is straightforward.

At this point, we reiterate that the proposed encryption is not secure. First and foremost, we would need to add some non-linear step in the process. Secondly, the fact that our primes are small is of concern, regardless if we omit certain primes and reorder them. However, the coding procedure may be applied to any preferred cryptosystem. Our only requirement is that the encrypted message be split into blocks, to create vectors.

\subsection{Coding Numbers Into Music}

We cease completing Example 4.4.1 to briefly discuss how we can actually create music starting with a vector $\vec{r}$ similar to the one on page 77 . There exist many programs 
that claim to convert numbers into music or sounds such as 'GoMinimal22' [26]. This program takes any text file and converts into a midi file. Moreover, it also does the conversion in the reverse direction. At first glance, using this program would seem to solve all of our problems. However, GoMinimal22 only helps us in one direction. When we converted the vector into a midi file we did obtain some 'music.' However, when we took the midi file to generate a text file, we did not obtain the original text file. Why? The reason is unknown since the decoding procedure is not given. Luckily for us, there does exist another freely available program that can help us: Csound [3].

With Csound, we are able to easily combine the music theory that we have already discussed with the large numbers we generate. The first step is to decide which pentatonic scale we are going to use. For simplicity, we use the five black keys, of the Gb pentatonic pitch collection, to better visualize. To identify each key on a piano, Csound uses the following notation: $x . y z$, where $x, y$ and $z$ are integers. The integer $x$ indicates which octave is being used. What is commonly known as 'middle $\mathrm{C}$ ' has $x=8$. Restricting ourselves to a single octave, we have twelve tones or keys. Each key is assigned an integer value between zero and eleven. Thus, $y$ is either zero or one and $z$ is defined as follows

$$
z=\left\{\begin{array}{l}
0,1, \ldots 9 \text { if } \mathrm{y}=0 \\
0 \text { or } 1 \text { if } y=1
\end{array}\right.
$$


Since we use the bass clef to carry the message, then $x \leq 7$. Thus, as we are using two pentatonic scales in the bass clef, we can easily differentiate these with Csound. If we use the two highest octaves of the bass clef, this means that $x=6$ and 7 . Therefore, we can describe the pentatonic scales by the numbers given in the following table.

\section{Table 4.5.1}

\begin{tabular}{|c|c|c|c|c|c|}
\hline Black keys $\rightarrow$ & $\mathrm{C} \sharp$ & $\mathrm{D} \sharp$ & $\mathrm{F} \sharp$ & $\mathrm{G} \sharp$ & $\mathrm{A} \sharp$ \\
\hline octave '1' $\rightarrow$ & 6.01 & 6.03 & 6.06 & 6.08 & 6.10 \\
\hline octave '2' $\rightarrow$ & 7.01 & 7.03 & 7.06 & 7.08 & 7.10 \\
\hline
\end{tabular}

We must also decide what to do with the minus sign, in the sense of how to map it. One way would be to cycle through the even and odd numbers. That is to say, the first positive number would have its ' + ' sign represented by zero, the next by two and so forth. Once we get to eight we start again from zero. Similarly, for the minus sign, we start from one, go to three up to nine and cycle back to one. Combining this with Tables 4.4.2 and 4.5.1 we obtain the following table. 
Table 4.5.2

\begin{tabular}{|c|c|c|c|c||c|c|c|c|c|}
\hline 6.01 & 6.03 & 6.06 & 6.08 & 6.10 & 7.01 & 7.03 & 7.06 & 7.08 & 7.10 \\
\hline$\nu_{0}$ & $\nu_{1}$ & $\nu_{2}$ & $\nu_{3}$ & $\nu_{4}$ & $\nu_{5}$ & $\nu_{6}$ & $\nu_{7}$ & $\nu_{8}$ & $\nu_{9}$ \\
\hline 7 & 1 & 6 & 3 & 0 & 8 & 9 & 2 & 4 & 5 \\
\hline- & - & + & - & + & + & - & + & + & - \\
\hline
\end{tabular}

We have transformed the vector on page 77 into its 'Csound representation', which is shown below. We note that the parentheses and slashes would not actually be present during this last transformation. The parentheses are there to help the reader differentiate between the actual number and postiveness/negativeness, while the slashes are there to distinguish the respective digits. We observe that the first number and only the first number of every line will have parentheses. Thus, in the first line, we first 'read' (6.10). Since 6.10 represents zero and zero is even, then this number is positive. We then look at what is left in the first string to determine that the number is +918475013750 .

$(6.10) 7.03 / 6.03 / 7.01 / 7.08 / 6.01 / 7.10 / 6.10 / 6.03 / 6.08 / 6.01 / 7.10 / 6.10$ (6.03)6.03/6.10/6.01/7.10/6.10/7.08/7.06/7.08/7.06/6.03/6.10/7.03/7.03 $(7.06) 6.08 / 7.01 / 6.06 / 7.03 / 6.06 / 7.08 / 7.03 / 7.01 / 7.08 / 6.01 / 7.10 / 7.01$ $(6.08) 7.06 / 7.06 / 6.03 / 6.08 / 7.03 / 6.06 / 6.06 / 7.01 / 7.01 / 6.03 / 6.08 / 7.03 / 6.08$ $(7.10) 6.03 / 6.03 / 6.10 / 6.10 / 7.08 / 6.01 / 7.06 / 6.01 / 6.08 / 6.01 / 6.01 / 7.10$ $(7.08) 7.01 / 7.10 / 6.03 / 7.06 / 6.03 / 6.03 / 6.03 / 6.03 / 7.01 / 6.06 / 7.10 / 7.01$ 
(6.01)6.03/7.01/7.01/7.10/6.03/6.06/6.06/7.10/6.03/7.10/7.06/6.08/7.10 (6.06)7.06/7.06/7.01/7.03/6.08/6.10/7.08/6.10/6.08/7.10/7.01/7.03/7.06 (7.03)6.03/7.06/6.03/6.03/6.01/7.03/6.03/6.01/6.08/7.08/6.06/6.10/6.06 (6.03)7.06/7.06/6.03/7.10/6.10/7.03/6.06/6.10/7.06/6.10/7.03/6.01/7.06 (7.01)7.06/7.01/7.06/7.08/7.03/7.08/6.01/6.03/7.01/7.08/7.06/6.06 (6.08)7.08/7.03/7.03/6.03/6.10/7.01/7.08/6.03/7.10/7.08/7.10/6.03/6.10 (6.10)6.01/6.06/6.01/7.01/7.03/6.03/6.06/7.01/6.08/6.10/6.03/6.08 (7.06)6.08/7.03/7.10/7.03/7.10/6.06/7.06/6.08/6.06/6.01/7.06/7.10/7.03 (7.10)6.03/7.06/6.10/6.10/6.10/6.01/7.06/7.01/7.03/6.06/7.10/7.10/6.10 (6.01)6.08/6.08/7.10/7.03/7.08/6.08/7.03/7.03/6.08/6.03/6.08/7.08/6.03 (7.08)6.03/6.06/6.10/7.03/6.10/6.01/7.03/7.08/7.03/6.06/6.10/6.06/7.03 (6.06)6.03/6.06/6.08/6.06/7.01/6.03/7.01/6.01/6.06/6.10/7.03/7.08 (7.03)6.03/7.01/6.01/6.03/7.10/7.06/6.08/6.03/6.08/7.01/7.06/7.08 (7.01)6.03/6.08/6.01/6.01/6.06/7.01/6.10/7.01/6.01/6.03/6.10/7.08/6.03 (6.10)6.03/7.03/7.06/7.10/7.03/6.03/7.03/6.08/6.08/6.10/6.08/7.06/6.06 $(7.06) 7.03 / 6.01 / 6.08 / 6.03 / 6.01 / 6.10 / 6.06 / 6.10 / 6.10 / 6.01 / 7.10 / 6.01$ $(6.03) 6.03 / 7.10 / 6.06 / 7.08 / 7.10 / 6.08 / 6.01 / 7.03 / 7.10 / 7.08 / 6.10 / 6.01 / 6.06$ (6.08)6.03/6.08/6.01/7.01/7.03/7.01/7.08/7.01/7.03/6.10/7.10/7.08 (7.10)6.03/7.01/7.03/6.10/6.01/7.01/6.10/6.01/7.06/7.01/6.01/6.01/7.10 (7.08)7.06/7.08/7.01/6.06/6.08/6.06/7.03/6.10/7.06/6.06/7.06 
(6.06)7.06/6.08/6.03/6.03/7.08/7.08/7.06/7.06/7.03/6.08/6.01/6.03

(7.01)7.08/7.06/7.03/6.01/7.10/7.01/6.03/7.08/6.08/7.01/7.01/7.08

(6.10)6.03/7.10/6.10/6.06/7.03/7.08/7.10/6.01/7.10/6.03/7.03/6.08/7.03

(7.06)7.06/6.03/7.08/7.08/6.06/6.08/7.01/7.01/6.01/7.10/7.10/7.06/7.10

(6.01)6.03/6.06/7.01/6.10/7.08/7.03/7.10/6.10/6.03/6.03/6.10/6.06/6.06

(7.03)7.10/6.08/7.10/6.08/6.08/6.06/7.01/6.10/7.03/6.08/6.10/6.03

We do not include the relevant Csound orchestra and score files in this thesis, due to the many pages it would take. However, we outline in some detail what we have done. The next step is to take these numbers and add extra 'fields' so that Csound knows what to do with the numbers. The instruments we choose decide how many fields we need. Aside from the type of instruments, we need to determine the number of instruments to be played simultaneously in the bass clef as well as the duration of each note. In our examples, we create four instruments that will simultaneously play the bass notes and we let the duration of each note be a tenth of a second. These instruments are 'named' i1, i2, i3 and i4. We further note that in our example the four instruments are identical, however, because of their names they are 'different' to Csound. Now, since the vector entries are not necessarily of the same length, there would be 'breaks' in the music. To embellish the music we then use a fifth instrument and as previously discussed, this instrument will play notes in higher octaves and will not carry any kind of information. This fifth instrument 
is denoted by $\mathrm{i} 5$.

The message 'This is a test.' has been made into a 12.7 seconds piece of music. Some may argue that this is too long for such a short message. However, we argue that we could have a message that is three times as long (in terms of the digit length of the message number) as our first message and would be approximately the same length musically. This is because we can have as many instruments as we want playing simultaneous notes. Each line of the score file begins by ix, where $x$ is some positive integer. For the bass notes in our example, we had $x=1,2,3$, and 4 . If it is assumed that we have at most sixteen notes per interval, then mathematically this means that the entries in the vector $\vec{r}$ could have a digit length of at most fifteen. As we computed in Example 4.4.1, this means that a message could have a total digit length of 384 . However, if instead of fifteen we have that the vector components of $\vec{r}$ have digit length of at most thirty-nine, then this implies that our message would then have a total digit length of at most $1152^{8}$ tripling the digit length. The trade-off however is that music will be 31.9 seconds long instead of 12.7 seconds, provided that only four instruments are used simultaneously for the bass notes. Should we also increase the number of instruments from four to eight, then instead of 31.9 seconds, the music would last for 15.9 seconds.

Now, how do we recover the message if notes are being played simultaneously?

\footnotetext{
${ }^{8}$ Keeping $n=32$, we solve $\left\lfloor\frac{\left(10^{16-1}-1\right) \times 2}{32 \times 33}\right\rfloor$.
} 
To recover the message we need only to look at the value of $x$ and the start time. The following example should help illustrate how.

Example 4.5.1 In our example, we have four instruments. Moreover, we know that we have at most sixteen notes per interval and that the duration of each tone is 0.1 seconds. Thus, each interval is a multiple of 1.6 . Now, suppose we want to recover the $n^{\text {th }}$ vector entry. The procedure is as follows.

1. Compute $n=4 q+r$, where $q$ and $r$ are positive integers and $0 \leq r \leq 3$.

2. Compute $1.6 \times(q-1)=k$. The value of $k$ indicates the start time of the interval of the desired vector entry.

3. The corresponding instrument is ir. If $r=0$, take $r$ to be four.

Hence, the fourth vector entry has a start time of 0 and is using instrument i4. The seventh entry has a start time of 1.6 and is using instrument i3. Finally, the thirty-second vector entry has a start time of 11.2 and is using instrument i4.

At this point, we summarize the steps involved to create our music. The following diagram should also be helpful. 


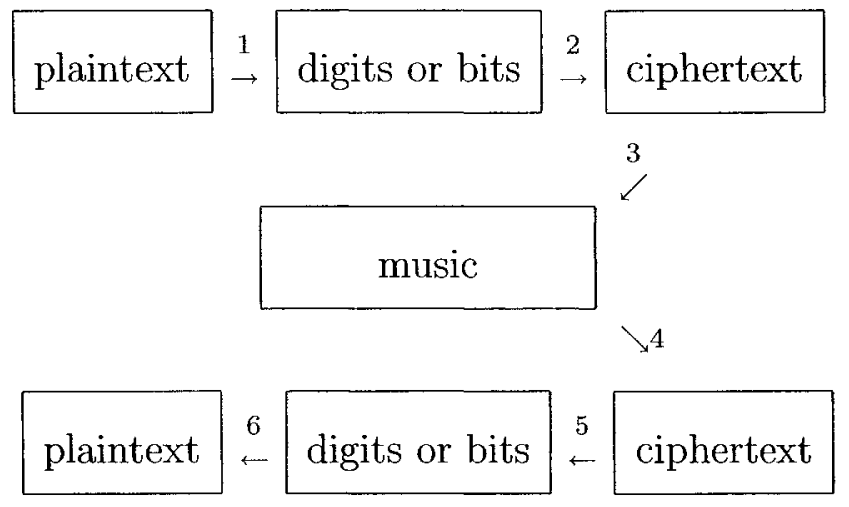

1. Encode the message into digits or bits.

2. Encrypt using preferred method of encryption, for example RSA.

3. Encode into music.

4. Decode the music into the ciphertext.

5. Decrypt to obtain string of digits or bits.

6. Decode to recover the original message.

We reiterate that our proposed encryption method is weak by today's standards. Determining the key size and adding a non-linear step are but a few problems to resolve. However, our coding process can take any encrypted message and convert it into music that 'makes sense'. 


\section{Chapter 5}

\section{Conclusions}

The goal of this thesis was to develop a procedure to encode messages in music without cacophony. We believe that this has been achieved, a feat that was initially thought to be very hard. Of course, we have only scratched the surface and much more can be done, both mathematically and musically.

From the mathematics point of view, one would have to enhance the cryptosystem's security, as previously discussed. While studying security issues, one would have to determine optimal values for the size of the matrices as well as the number of notes per interval. We used $32 \times 32$ matrices, but is this best? Do we actually require bounds, upper and or lower? As for the number of notes per interval, do we want the interval to be four seconds? Again, do we actually require bounds? It would also be interesting and worthwhile to examine if other types of mathematics 
can be used in a similar fashion, such as Abstract Algebra or even Calculus. There is also the issue of data compression. Is there a way to reduce the "message number $m$ ' so that the music have a shorter duration? Lastly, on page 38 we mentioned that we could not verify which pair of matrices $C$ and $M$ are being used. Can one determine the number of such factorizations in polynomial time? Is the number of factorizations infinite? If we want the number of factorizations to be infinite, one could consider having $C$ to be a non-square matrix with more columns than rows. This creates the new problem of how would we recover $M$ (or $\vec{m}$ if we consider $M$ to be $n \times 1)$ ?

Other problems that should be addressed with respect to music are the following. As discussed in Chapter 4, one could make use of accents. We have used the pentatonic scale, however, other scales do exist. Investigating them could provide a way to generate perhaps more traditional music or mainstream music. Additionally, with Csound, one can design instruments. If we are to continue using Csound, it would be most beneficial to create instruments that actually simulate existing instruments such as pianos, guitars or even say saxophones. Perhaps the more difficult aspect of the music is that the determination of what is 'pleasant' is always a subjective aesthetic judgement. After all, what one considers to be enjoyable another may judge to be most unpleasant.

Indeed there is a lot to accomplish. However, the groundwork, we feel, has been 
laid. Mathematics and music are dynamic fields of study. In light of the continuous growth in technology, both areas of research will exploit all relevant advancements and developments. We end our current discussion with a few quotes [20].

We used to think that if we knew one, we knew two, because one and one are two. We are finding that we must learn a great deal more about 'and'.

- Sir Arthur Eddington, English astronomer (1882 - 1944)

The whole problem can be stated quite simply by asking, 'Is there a meaning to music?' My answer would be, 'Yes.' And 'Can you state in so many words what the meaning is?' My answer to that would be, 'No.' - Aaron Copland, US composer (1900 - 1990) 


\section{Bibliography}

[1] George E. Andrews. Number Theory Dover Publications, 1994.

[2] Eric Blom (editor). Cryptography, Musical. Grove's Dictionary of Music and Musicians, $5^{\text {th }}$ ed. Macmillan Press Limited, 1973.

[3] Richard Boulager (editor). The Csound Book: Perspective in Software Synthesis, Sound Design, Signal Processing, and Programming. The MIT Press, 2000.

[4] Lindsay N. Childs. A Concrete Introduction to Higher Algebra, $2^{\text {nd }}$ ed. Springer, 2000.

[5] Allen Forte. The Structure of Atonal Music. Yale University Press, 1973.

[6] Joachim von zur Gathen and Jürgen Gerhard. Modern Computer Algebra, $2^{\text {nd }}$ ed. Cambridge University Press, 2003.

[7] Michael Miller. The Complete Idiot's Guide to Music Theory. Penguin Group (USA) Inc., 2002.

[8] Ernest Nagel and James R. Newman. Gödel's Proof. $7^{\text {th }}$ impression, New York University Press, 1968.

[9] Stanley Sadie (editor). Cryptography, Musical. The New Grove Dictionary of Music and Musicians, Macmillan Publishers Limited, 1980.

[10] Stanley Sadie (editor). Triad. The New Grove Dictionary of Music and Musicians, Macmillan Publishers Limited, 1980.

[11] Simon Singh. The Code Book: The Science of Secrecy From Ancient Egypt to Quantum Cryptography. Anchor Books (A Division of Random House Inc.), 1999.

[12] Joseph N. Straus. Introduction to Post-Tonal Theory. $3^{\text {rd }}$ ed., Prentice Hall, 2005. 


\section{Webpages}

[13] ACME LABS. Bigint: Large Integer Package. $\quad 7 \quad$ June 2006 <http://www.acme.com/software/bigint/>.

[14] ASCII. ASCII HTML Codes. 2001. 30 May $2006<$ http://www.ascii.cl/htmlcodes.htm>.

[15] John Boyd-Brent Harmony and Proposition: Pythagoras: Music and Space. 1995. 14 May $2006<$ http://aboutscotland.co.uk/harmony/prop.html $>$.

[16] Daniel Cummerow. The Sound of Mathematics. 1998.14 May 2006 <http://www.geocities.com/Vienna/9349/index.html>.

[17] Xiao Gang (site Manager). WWW Interactive Multipurpose Server. 1997. WIMS. 7 June $2006<$ http://wims.unice.fr/wims/wims.cgi?lang $=$ en $\&+$ session=BX712AB446.1>.

[18] Gary C. Kessler. Steganography: Hiding Data Within Data. 2001. 5 June 2006 <http://www.garykessler.net/library/steganography.html>.

[19] Merriam-Webster. Merriam-Webster Online Dictionary. 2006. Merriam-Webster Incorporated. 8 August $2006<$ http://www.m-w.com/dictionary/music $>$.

[20] Michael Moncur. The Quotations Page. 1994. The Quotations Page. 22 June 2006 $<$ http://www.quotationspage.com/>.

[21] Thomas (Penn) Leary. Cryptology in the 16th and 17th Centuries. Cryptologia: A quarterly journal devoted to Cryptology. 1996. Taylor \& Francis. 14 May 2006 <http://home.att.net/ tleary/cryptolo.htm>.

[22] National Geographic Kids. Secret Messages and Disappearing Ink Craft. 1996. National Geographic. 17 May 2006 <http://www.nationalgeographic.com/ngkids/trythis/ghostly_messages.html>.

[23] Fabien A. P. Petitcolas. $\quad$ mp3stego. $\quad 1997 . \quad 14 \quad$ May 2006 <http://www.petitcolas.net/fabien/steganography/mp3stego/

[24] Fabien A. P. Petrtcolas. $\quad$ mp3stego. $\quad$ 1997. 14 May 2006 <http://www.petitcolas.net/fabien/steganography/mp3stego/svega.mp3

[25] Fabien A. P. Pettacolas. $\quad$ mp3stego. $\quad 1997 . \quad 14 \quad$ May 2006 <http://www.petitcolas.net/fabien/steganography/mp3stego/svega_stego.mp3

[26] Proletsoft. Proletsoft's GoMinimal! v2.2. 2006. Proletsoft. 22 June 2006 <http://proletsoft.freeservers.com/mmb/gominimal.html>.

[27] Miller PucketTe. 19 May $2006<$ http://crca.ucsd.edu/ msp/index.htm>. 
[28] Patrick J. Russell, JR (COMPILER). Books on Cryptography from the Arnold Semeiology Collection. 1993. Washington University in St.Louis. 15 May 2006 $<$ http://library.wustl.edu/units/spec/rarebooks/semeiology/cryptography.html>.

[29] University of London. RSA Basics. Royal Holloway, University of London, Department of Computer Science. 5 June 2006 $<$ http://www.cs.rhul.ac.uk/home/fionn/qub/csc317/x5.pdf >.

[30] Kristy Westphal. Steganography Revealed. 2003. Security Focus. 14 May 2006 $<$ http://www.securityfocus.com/infocus/1684>.

[31] WiKIPEDIA. ASCII. 2006. 14 May $2006<$ http://en.wikipedia.org/wiki/ASCII>.

[32] Wikipedia. Pentatonic Scale. 2006. 16 May 2006 $<$ http://en.wikipedia.org/wiki/Pentatonic $>$.

[33] WIKIPEdiA. Allen Forte. 2006. 16 May $2006<$ http://en.wikipedia.org/wiki/Allen_Forte>.

[34] Wikipedia. Circle of Fifths. 2006. 17 May 2006 $<$ http://en.wikipedia.org/wiki/Circle_of_fifths $>$.

[35] WiKIPEDIA. Steganography. 2006. $17 \quad$ May 2006 $<$ http://en.wikipedia.org/wiki/Steganography>. 